\title{
Planck early results. XX. New light on anomalous microwave emission from spinning dust grains ${ }^{\star}$
}

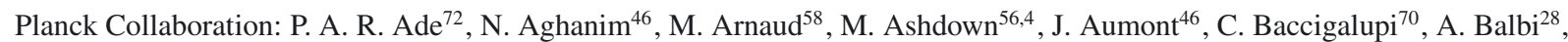
A. J. Banday ${ }^{77,7,63}$, R. B. Barreiro ${ }^{52}$, J. G. Bartlett ${ }^{3,54}$, E. Battaner ${ }^{79}$, K. Benabed ${ }^{47}$, A. Benoît ${ }^{45}$, J.-P. Bernard ${ }^{77,7}$, M. Bersanelli ${ }^{25,41}$, R. Bhatia ${ }^{5}$, J. J. Bock ${ }^{54,8}$, A. Bonaldi ${ }^{37}$, J. R. Bond ${ }^{6}$, J. Borrill ${ }^{62,73}$, F. R. Bouchet ${ }^{47}$, F. Boulanger ${ }^{46}$, M. Bucher ${ }^{3}$, C. Burigana ${ }^{40}$, P. Cabella ${ }^{28}$, B. Cappellini ${ }^{41}$, J.-F. Cardoso ${ }^{59,3,47}$, S. Casassus ${ }^{76}$, A. Catalano ${ }^{3,57}$, L. Cayón ${ }^{18}$, A. Challinor ${ }^{49,56,10}$, A. Chamballu ${ }^{43}$, R.-R. Chary ${ }^{44}$, X. Chen ${ }^{44}$, L.-Y. Chiang ${ }^{48}$,

C. Chiang ${ }^{17}$, P. R. Christensen ${ }^{67,29}$, D. L. Clements ${ }^{43}$, S. Colombi ${ }^{47}$, F. Couchot ${ }^{61}$, A. Coulais ${ }^{57}$, B. P. Crill ${ }^{54,68}$, F. Cuttaia ${ }^{40}$, L. Danese ${ }^{70}$,

R. D. Davies ${ }^{55}$, R. J. Davis ${ }^{55}$, P. de Bernardis ${ }^{24}$, G. de Gasperis ${ }^{28}$, A. de Rosa ${ }^{40}$, G. de Zotti ${ }^{37,70}$, J. Delabrouille ${ }^{3}$, J.-M. Delouis ${ }^{47}$, C. Dickinson ${ }^{55}$, S. Donzelli ${ }^{41,50}$, O. Doré ${ }^{54,8}$, U. Dörl ${ }^{63}$, M. Douspis ${ }^{46}$, X. Dupac ${ }^{32}$, G. Efstathiou ${ }^{49}$, T. A. Enßlin ${ }^{63}$, H. K. Eriksen ${ }^{50}$, F. Finelli $^{40}$, O. Forni ${ }^{77,7}$,

M. Frailis ${ }^{39}$, E. Franceschi ${ }^{40}$, S. Galeotta ${ }^{39}$, K. Ganga ${ }^{3,44}$, R. T. Génova-Santos ${ }^{51,30}$, M. Giard ${ }^{77,7}$, G. Giardino ${ }^{33}$, Y. Giraud-Héraud ${ }^{3}$,

J. González-Nuevo ${ }^{70}$, K. M. Górski ${ }^{4,81}$, S. Gratton ${ }^{56,49}$, A. Gregorio ${ }^{26}$, A. Gruppuso ${ }^{40}$, F. K. Hansen ${ }^{50}$, D. Harrison ${ }^{49,56}$, G. Helou $^{8}$,

S. Henrot-Versillé61 , D. Herranz ${ }^{52}$, S. R. Hildebrandt ${ }^{8,60,51}$, E. Hivon ${ }^{47}$, M. Hobson ${ }^{4}$, W. A. Holmes ${ }^{54}$, W. Hovest ${ }^{63}$, R. J. Hoyland ${ }^{51}$,

K. M. Huffenberger ${ }^{80}$, T. R. Jaffe ${ }^{77,7}$, A. H. Jaffe ${ }^{43}$, W. C. Jones ${ }^{17}$, M. Juvela ${ }^{16}$, E. Keihänen ${ }^{16}$, R. Keskitalo ${ }^{54,16}$, T. S. Kisner ${ }^{62}$, R. Kneissl ${ }^{31,5}$,

L. Knox ${ }^{20}$, H. Kurki-Suonio ${ }^{16,35}$, G. Lagache ${ }^{46}$, A. Lähteenmäki ${ }^{1,35}$, J.-M. Lamarre ${ }^{57}$, A. Lasenby ${ }^{4,56}$, R. J. Laureijs ${ }^{33}$, C. R. Lawrence ${ }^{54}$,

S. Leach ${ }^{70}$, R. Leonardi ${ }^{32,33,21}$, P. B. Lilje ${ }^{50,9}$, M. Linden-Vørnle ${ }^{12}$, M. López-Caniego ${ }^{52}$, P. M. Lubin ${ }^{21}$, J. F. Macías-Pérez ${ }^{60}$, C. J. MacTavish ${ }^{56}$,

B. Maffei ${ }^{55}$, D. Maino ${ }^{25,41}$, N. Mandolesi ${ }^{40}$, R. Mann ${ }^{71}$, M. Maris ${ }^{39}$, D. J. Marshall ${ }^{77,7}$, E. Martínez-González ${ }^{52}$, S. Masi $^{24}$, S. Matarrese ${ }^{23}$,

F. Matthai ${ }^{63}$, P. Mazzotta ${ }^{28}$, P. McGehee ${ }^{44}$, P. R. Meinhold ${ }^{21}$, A. Melchiorri ${ }^{24}$, L. Mendes ${ }^{32}$, A. Mennella ${ }^{25,39}$, S. Mitra ${ }^{54}$,

M.-A. Miville-Deschênes ${ }^{46,6}$, A. Moneti ${ }^{47}$, L. Montier ${ }^{77,7}$, G. Morgante ${ }^{40}$, D. Mortlock ${ }^{43}$, D. Munshi ${ }^{72,49}$, A. Murphy ${ }^{66}$, P. Naselsky ${ }^{67,29}$, P. Natoli ${ }^{27,2,40}$, C. B. Netterfield ${ }^{14}$, H. U. Nørgaard-Nielsen ${ }^{12}$, F. Noviello ${ }^{46}$, D. Novikov ${ }^{43}$, I. Novikov ${ }^{67}$, I. J. O' Dwyer ${ }^{54}$, S. Osborne ${ }^{75}$, F. Pajot ${ }^{46}$, R. Paladini ${ }^{74,8}$, B. Partridge ${ }^{34}$, F. Pasian ${ }^{39}$, G. Patanchon ${ }^{3}$, T. J. Pearson ${ }^{8,44}$, M. Peel ${ }^{55}$, O. Perdereau ${ }^{61}$, L. Perotto ${ }^{60}$, F. Perrotta ${ }^{70}$, F. Piacentini ${ }^{24}$, M. Piat ${ }^{3}$, S. Plaszczynski ${ }^{61}$, P. Platania ${ }^{53}$, E. Pointecouteau ${ }^{77,7}$, G. Polenta ${ }^{2,38}$, N. Ponthieu ${ }^{46}$, T. Poutanen ${ }^{35,16,1}$, G. Prézeau $^{8,54}$, P. Procopio $^{40}$, S. Prunet ${ }^{47}$, J.-L. Puget ${ }^{46}$, W. T. Reach ${ }^{78}$, R. Rebolo ${ }^{51,30}$, W. Reich ${ }^{64}$, M. Reinecke ${ }^{63}$, C. Renault ${ }^{60}$, S. Ricciardi ${ }^{40}$, T. Riller ${ }^{63}$, I. Ristorcelli ${ }^{77,7}$, G. Rocha ${ }^{54,8}$, C. Rosset $^{3}$, M. Rowan-Robinson ${ }^{43}$, J. A. Rubiño-Martín ${ }^{51,30}$, B. Rusholme ${ }^{44}$, M. Sandri ${ }^{40}$, D. Santos ${ }^{60}$, G. Savini $^{69}$, D. Scott ${ }^{15}$, M. D. Seiffert ${ }^{5,8}$, P. Shellard ${ }^{10}$, G. F. Smoot ${ }^{19,62,3}$, J.-L. Starck ${ }^{58,11}$, F. Stivoli ${ }^{42}$, V. Stolyarov ${ }^{4}$, R. Stompor ${ }^{3}$, R. Sudiwala ${ }^{72}$, J.-F. Sygnet ${ }^{47}$, J. A. Tauber ${ }^{33}$, L. Terenzi ${ }^{40}$, L. Toffolatti ${ }^{13}$, M. Tomasi ${ }^{25,41}$, J.-P. Torre ${ }^{46}$, M. Tristram ${ }^{61}$, J. Tuovinen ${ }^{65}$, G. Umana ${ }^{36}$, L. Valenziano $^{40}$, J. Varis $^{65}$,

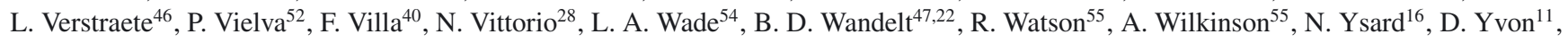
A. Zacchei ${ }^{39}$, and A. Zonca ${ }^{21}$

(Affiliations can be found after the references)

Received 8 January 2011 / Accepted 11 May 2011

\begin{abstract}
Anomalous microwave emission (AME) has been observed by numerous experiments in the frequency range $\sim 10-60 \mathrm{GHz}$. Using Planck maps and multi-frequency ancillary data, we have constructed spectra for two known AME regions: the Perseus and $\rho$ Ophiuchi molecular clouds. The spectra are well fitted by a combination of free-free radiation, cosmic microwave background, thermal dust, and electric dipole radiation from small spinning dust grains. The spinning dust spectra are the most precisely measured to date, and show the high frequency side clearly for the first time. The spectra have a peak in the range $20-40 \mathrm{GHz}$ and are detected at high significances of $17.1 \sigma$ for Perseus and 8.4 $\sigma$ for $\rho$ Ophiuchi. In Perseus, spinning dust in the dense molecular gas can account for most of the AME; the low density atomic gas appears to play a minor role. In $\rho$ Ophiuchi, the $\sim 30 \mathrm{GHz}$ peak is dominated by dense molecular gas, but there is an indication of an extended tail at frequencies 50-100 GHz, which can be accounted for by irradiated low density atomic gas. The dust parameters are consistent with those derived from other measurements. We have also searched the Planck map at $28.5 \mathrm{GHz}$ for candidate AME regions, by subtracting a simple model of the synchrotron, free-free, and thermal dust. We present spectra for two of the candidates; S140 and S235 are bright H II regions that show evidence for AME, and are well fitted by spinning dust models.
\end{abstract}

Key words. ISM: general - Galaxy: general - radiation mechanisms: general - radio continuum: ISM - submillimeter: ISM

\section{Introduction}

Anomalous microwave emission (AME) is an additional component of diffuse foreground emission that cannot be easily explained by synchrotron, free-free, or thermal dust emission.

^ Corresponding author: C. Dickinson,

e-mail: Clive.Dickinson@manchester.ac.uk
AME has been observed by numerous experiments over the frequency range $\sim 10-60 \mathrm{GHz}$ and is found to be very closely correlated with far infrared (FIR) emission associated with thermal emission from dust grains (Kogut et al. 1996; Leitch et al. 1997; de Oliveira-Costa et al. 1997; Banday et al. 2003; Lagache 2003; de Oliveira-Costa et al. 2004; Finkbeiner 2004; Finkbeiner et al. 2004; Davies et al. 2006; Dobler \& Finkbeiner 2008; 
Miville-Deschênes et al. 2008; Gold et al. 2011; Ysard et al. 2010). Electric dipole radiation from small rapidly spinning dust grains, or "spinning dust", is thought to be emitted in the microwave region of the spectrum ${ }^{1}$. Theoretical models predict a peaked spectrum, at a range of possible frequencies $(\sim 10-150 \mathrm{GHz})$ depending on the properties of the dust grains and their environment (Draine \& Lazarian 1998; Ali-Haïmoud et al. 2009; Dobler et al. 2009; Ysard \& Verstraete 2010). The spectrum is very different from those of the traditional diffuse emission expected at these frequency ranges; for example, free-free, synchrotron, and thermal dust emission have powerlaw-like spectra at microwave frequencies. For these reasons, spinning dust emission has become the best explanation for the AME, although other physical mechanisms could still be contributing at some level, such as hot free-free emission (Leitch et al. 1997), hard synchrotron radiation (Bennett et al. 2003), or magneto-dipole emission (Draine \& Lazarian 1999).

The most direct evidence for spinning dust grains has come from dedicated observations of known dust clouds in the interstellar medium (Finkbeiner et al. 2002; Casassus et al. 2006; Dickinson et al. 2009; Scaife et al. 2009; Dickinson et al. 2009, 2010). Perhaps the best example is that of the Perseus molecular cloud. Watson et al. (2005) observed the region with the COSMOSOMAS telescope covering 11-17 GHz. By combining data from radio surveys, WMAP, and DIRBE, they were able to show very clearly a strong excess at frequencies $\sim 10-60 \mathrm{GHz}$ and a shape similar to spinning dust models. Another clear example is that of the photodissociation region (PDR) associated with the $\rho$ Ophiuchi molecular dust cloud. Casassus et al. (2008) observed the region centred at $(l, b)=\left(353^{\circ} .0,+17^{\circ} .0\right)$ at $31 \mathrm{GHz}$ with the Cosmic Background Imager (CBI) and found a close connection between the centimetre-wavelength emission and the dust emission. Further analysis of the spectrum confirmed that there was significant excess emission that was consistent with spinning dust models. AME has also been detected within a starforming region in the galaxy NGC 6946 (Murphy et al. 2010), which appears to be well-fitted by spinning dust models (Scaife et al. 2010).

Planck $^{2}$ data now represent the next step in measuring and understanding spinning dust emission. The additional data at 28.5, 44.1, and $70.3 \mathrm{GHz}$ (where spinning dust is expected to emit strongly) and the high frequency data at $100-857 \mathrm{GHz}$ allow an accurate model for the thermal emission to be removed. This is crucial in defining a precise spectrum for the spinning dust, particularly at the higher frequencies where the smallest dust grains dominate. Accurate spectra for spinning dust could potentially prove to be an important diagnostic tool for measuring the properties of the dust (e.g., the size distribution, density and average electric dipole moment) as well as the physical environment in the surrounding region (e.g., the interstellar radiation field).

In this paper, we study two known AME regions, the Perseus and $\rho$ Ophiuchi molecular cloud regions, and provide spectra over the frequency range $0.4-3000 \mathrm{GHz}$. We show, for the first time, an accurate residual spectrum after removal of the freefree, cosmic microwave background (CMB), and thermal dust

\footnotetext{
1 Erickson (1957) was the first to suggest the possibility of radio emission from spinning dust grains.

2 Planck (http://WwW.esa.int/Planck) is a project of the European Space Agency (ESA) with instruments provided by two scientific consortia funded by ESA member states (in particular the lead countries France and Italy), with contributions from NASA (USA) and telescope reflectors provided by a collaboration between ESA and a scientific consortium led and funded by Denmark.
}

components. The AME is clearly detected as a convex spectrum that is well-fitted by theoretical models of spinning dust emission. We investigate the parameters for the spinning dust to see whether they are reasonable, given the conditions in these environments. The Planck data, in combination with ancillary data, are also used to search for new regions of AME. We present two such regions and show that these newly discovered regions can also be fitted with a contribution from spinning dust grains.

In this paper, Sects. 2 and 3 describe the Planck and ancillary data that were used to measure the spectra of AME regions. Section 4 presents the results for the Perseus molecular cloud and Sect. 5 for the $\rho$ Ophiuchi cloud. Section 6 describes preliminary modelling of the AME in terms of spinning dust. Section 7 presents the search for new AME regions and gives two clear examples. Conclusions are given in Sect. 8.

\section{Planck data}

Planck (Tauber et al. 2010; Planck Collaboration 2011a) is the third-generation space mission to measure the anisotropy of the cosmic microwave background (CMB). It observes the sky in nine frequency bands covering $30-857 \mathrm{GHz}$ with high sensitivity and angular resolution from $31^{\prime}$ to $5^{\prime}$. The Low Frequency Instrument (LFI; Mandolesi et al. 2010; Bersanelli et al. 2010; Mennella et al. 2011) covers the 30, 44, and $70 \mathrm{GHz}$ bands with amplifiers cooled to $20 \mathrm{~K}$. The High Frequency Instrument (HFI; Lamarre et al. 2010; Planck HFI Core Team 2011a) covers the $100,143,217,353,545$, and $857 \mathrm{GHz}$ bands with bolometers cooled to $0.1 \mathrm{~K}$. Polarisation is measured in all but the highest two bands (Leahy et al. 2010; Rosset et al. 2010). A combination of radiative cooling and three mechanical coolers produces the temperatures needed for the detectors and optics (Planck Collaboration 2011b). Two data processing centres (DPCs) check and calibrate the data and make maps of the sky (Planck HFI Core Team 2011b; Zacchei et al. 2011). Planck's sensitivity, angular resolution, and frequency coverage make it a powerful instrument for Galactic and extragalactic astrophysics as well as cosmology. Early astrophysics results are given in Planck Collaboration $(2011 \mathrm{~h}-\mathrm{z})$.

In this paper, we start with the Planck (non CMB-subtracted) maps at nominal frequencies of 28.5, 40.1, 70.3, 100, 143, 217, 353,545 , and $857 \mathrm{GHz}$ (see Table 1). For the spectral analyses, we smoothed the HEALPix maps to a common angular resolution of $1^{\circ}$ and degraded to $N_{\text {side }}=512$ (Górski et al. 2005). We assume Gaussian beams and use the average beamwidths given in Planck HFI Core Team (2011b); Zacchei et al. (2011). Details of the beam, such as the exact shape and variation of the beam across the sky, do not significantly affect the results presented here, due to the applied smoothing which dominates the spatial response of the maps. Furthermore, extended emission is less sensitive to the details of the beam. We convert from CMB thermodynamic units to Rayleigh-Jeans brightness temperature units using the standard conversion factors given in Planck HFI Core Team (2011b) and Zacchei et al. (2011). Colour corrections to account for the finite bandpass at each frequency are applied during the modelling of the spectra for each object; these are typically a few per cent for LFI and $10-15 \%$ for HFI. The $100 \mathrm{GHz}$ data are significantly contaminated by the $\mathrm{CO} J=1 \rightarrow 0$ line at $115 \mathrm{GHz}$. We apply a nominal correction to the $100 \mathrm{GHz}$ map using the CO survey of Dame et al. (2001). We multiply the integrated $1^{\circ}$-smoothed line intensity map (in units of $\mathrm{K} \mathrm{km} \mathrm{s}^{-1}$ ) by 14.2 (Planck HFI Core Team 2011b) and subtract it from the $100 \mathrm{GHz}$ map. Instead of attempting to correct for other lines that may contaminate the 217 and $353 \mathrm{GHz}$ data, we do 
Table 1. Summary of the data.

\begin{tabular}{|c|c|c|c|c|}
\hline $\begin{array}{l}\text { Frequency } \\
{[\mathrm{GHz}]}\end{array}$ & $\begin{array}{c}\text { Telescope/ } \\
\text { survey }\end{array}$ & $\begin{array}{c}\text { Angular resolution } \\
\text { [arcmin] }\end{array}$ & Coverage & Notes \\
\hline 0.408 & JB/Eff/Parkes ${ }^{a}$ & $\approx 60$ & Full-sky & NCSA desourced/destriped version ${ }^{b}$ \\
\hline 0.820 & Dwingeloo $^{c}$ & 72 & Northern sky $\left(-7^{\circ}<\delta<+85^{\circ}\right)$ & Available on the $\mathrm{web}^{d}$ \\
\hline 1.420 & Stockert/Villa-Elisa ${ }^{e}$ & 36 & Full-sky & Courtesy of Reich \\
\hline 2.326 & HartRAO $^{f}$ & 20 & Southern sky $\left(-83^{\circ}<\delta<+13 / 32^{\circ}\right)$ & Courtesy of Jonas (priv. comm.) \\
\hline $11-17$ & COSMOSOMAS $^{g}$ & $\approx 60$ & Northern sky $\left(+24^{\circ}<\delta<+44^{\circ}\right)$ & Filtered on large angular scales \\
\hline 22.8 & WMAP 7-yr ${ }^{h}$ & $\approx 49$ & Full-sky & $1^{\circ}$ smoothed version ${ }^{b}$ \\
\hline 28.5 & Planck $\mathrm{LFI}^{i}$ & $32: 65$ & Full-sky & \\
\hline 33.0 & WMAP 7-yr ${ }^{h}$ & $\approx 40$ & Full-sky & $1^{\circ}$ smoothed version $^{b}$ \\
\hline 40.7 & WMAP 7-yr ${ }^{h}$ & $\approx 31$ & Full-sky & $1^{\circ}$ smoothed version $^{b}$ \\
\hline 44.1 & Planck $\mathrm{LFI}^{i}$ & $27 ! 92$ & Full-sky & \\
\hline 60.7 & WMAP 7-yr ${ }^{h}$ & $\approx 21$ & Full-sky & $1^{\circ}$ smoothed version $^{b}$ \\
\hline 70.3 & Planck $\mathrm{LFI}^{i}$ & $13 ! 01$ & Full-sky & \\
\hline 93.5 & WMAP 7-yr ${ }^{h}$ & $\approx 13$ & Full-sky & $1^{\circ}$ smoothed version ${ }^{b}$ \\
\hline 100 & Planck $\mathrm{HFI}^{j}$ & $9 ! 37$ & Full-sky & \\
\hline 143 & Planck $\mathrm{HFI}^{j}$ & $7 ! .04$ & Full-sky & \\
\hline 217 & Planck $\mathrm{HFI}^{j}$ & 4.68 & Full-sky & \\
\hline 353 & Planck $\mathrm{HFI}^{j}$ & 4.43 & Full-sky & \\
\hline 545 & Planck $\mathrm{HFI}^{j}$ & $3: 80$ & Full-sky & \\
\hline 857 & Planck $\mathrm{HFI}^{j}$ & 3.67 & Full-sky & \\
\hline 1249 & COBE-DIRBE $^{k}$ & $\approx 40$ & Full-sky & LAMBDA website ${ }^{b}$ \\
\hline 2141 & COBE-DIRBE $^{k}$ & $\approx 40$ & Full-sky & LAMBDA website ${ }^{b}$ \\
\hline 2997 & COBE-DIRBE $^{k}$ & $\approx 40$ & Full-sky & LAMBDA website $^{b}$ \\
\hline
\end{tabular}

Notes. ${ }^{(a)}$ Haslam et al. (1982); (b) http://lambda.gsfc.nasa.gov/; (c) Berkhuijsen (1972); (d) http://www.mpifr-bonn.mpg.de/ survey.html; (e) Reich (1982); Reich \& Reich (1986); Reich et al. (2001); ${ }^{(f)}$ Jonas et al. (1998); (g) Watson et al. (2005); (h) Jarosik et al. (2011); ${ }^{(i)}$ Zacchei et al. (2011); ${ }^{(j)}$ Planck HFI Core Team (2011b); ${ }^{(k)}$ Hauser et al. (1998).

not include these channels in the modelling of spectra if they are observed to be in excess by more than $2 \sigma$, relative to the best-fit model when omitting these frequencies. Uncertainties are taken to be the nominal uncertainties in absolute calibration and gain (given in the aforementioned papers), which are added in quadrature. If these are below $3 \%$, we conservatively assume an overall uncertainty of $3 \%$.

Jack-knife tests, in which the data were split into two halves (in terms of time and also HFI detectors; see Planck HFI Core Team 2011b), provided a check of the consistency of the Planck data. The tests confirmed that the results were consistent with each other to within a small fraction of the derived uncertainties.

\section{Ancillary data}

Although Planck observes over a wide frequency range (30-857 GHz), it is still important to include ancillary data to extend the frequency range into the FIR, and in particular, to lower frequencies. To supplement Planck data we therefore use a range of publicly available surveys and data available within the team. Table 1 summarises the data used in the analysis and the origin of the maps that were used. In the following sections, we describe the various data sets in more detail.

\subsection{Low frequency radio data}

The radio data consist of a number of low frequency surveys in the range $0.408-2.326 \mathrm{GHz}$ (Table 1). We used the most up-todate versions of the data sets, and where necessary, regridded the maps into the HEALPix format (Górski et al. 2005) by using a procedure which computes the surface intersection between individual pixels of the survey with the intersecting HEALPix pixels. This procedure has been shown to conserve photometry (Paradis et al., in prep.). We note that there are significant baseline uncertainties (i.e., offsets) in these maps, but these will not affect our results since we are subtracting a local background for each object, which removes any such offset. The data include the full-sky map at $408 \mathrm{MHz}$ (Haslam et al. 1982), the $820 \mathrm{MHz}$ Dwingeloo survey (Berkhuijsen 1972), the new full-sky map at 1420 MHz (Reich 1982; Reich \& Reich 1986; Reich et al. 2001), and the $2326 \mathrm{MHz}$ HartRAO southern survey (Jonas et al. 1998). The $1420 \mathrm{MHz}$ map was multiplied by a factor of 1.55 to account for the main beam to full beam ratio (Reich \& Reich 1988), and to bring the calibration in line with the other data. We assume a $10 \%$ overall calibration uncertainty for these data.

\subsection{COSMOSOMAS data}

The COSMOSOMAS experiments (Gallegos et al. 2001) consisted of two circular scanning instruments COSMO11 (Hildebrandt et al. 2007) and COSMO15 (Fernández-Cerezo et al. 2006) which operated at the Teide observatory (altitude $2400 \mathrm{~m}$, Tenerife). They have produced $0.8-1.1$ resolution maps of $\approx 10000 \mathrm{deg}^{2}$ in four frequency bands $(10.9,12.7,14.7$, $16.3 \mathrm{GHz}$ ) that are ideal for filling the gap between low frequency radio surveys and the lowest frequency channels of WMAP and Planck. The average sensitivity of each map is in the range $80-120 \mu \mathrm{K}$ per beam, and the observing strategy is described in Gallegos et al. (2001). As COSMOSOMAS was a ground-based experiment, there is significant contamination from atmospheric and receiver $1 / f$ noise, which needs to be filtered out. This results in a loss of response to angular scales larger than $5^{\circ}$ and introduces low level correlations over the scan ring. This must be taken into account when considering 

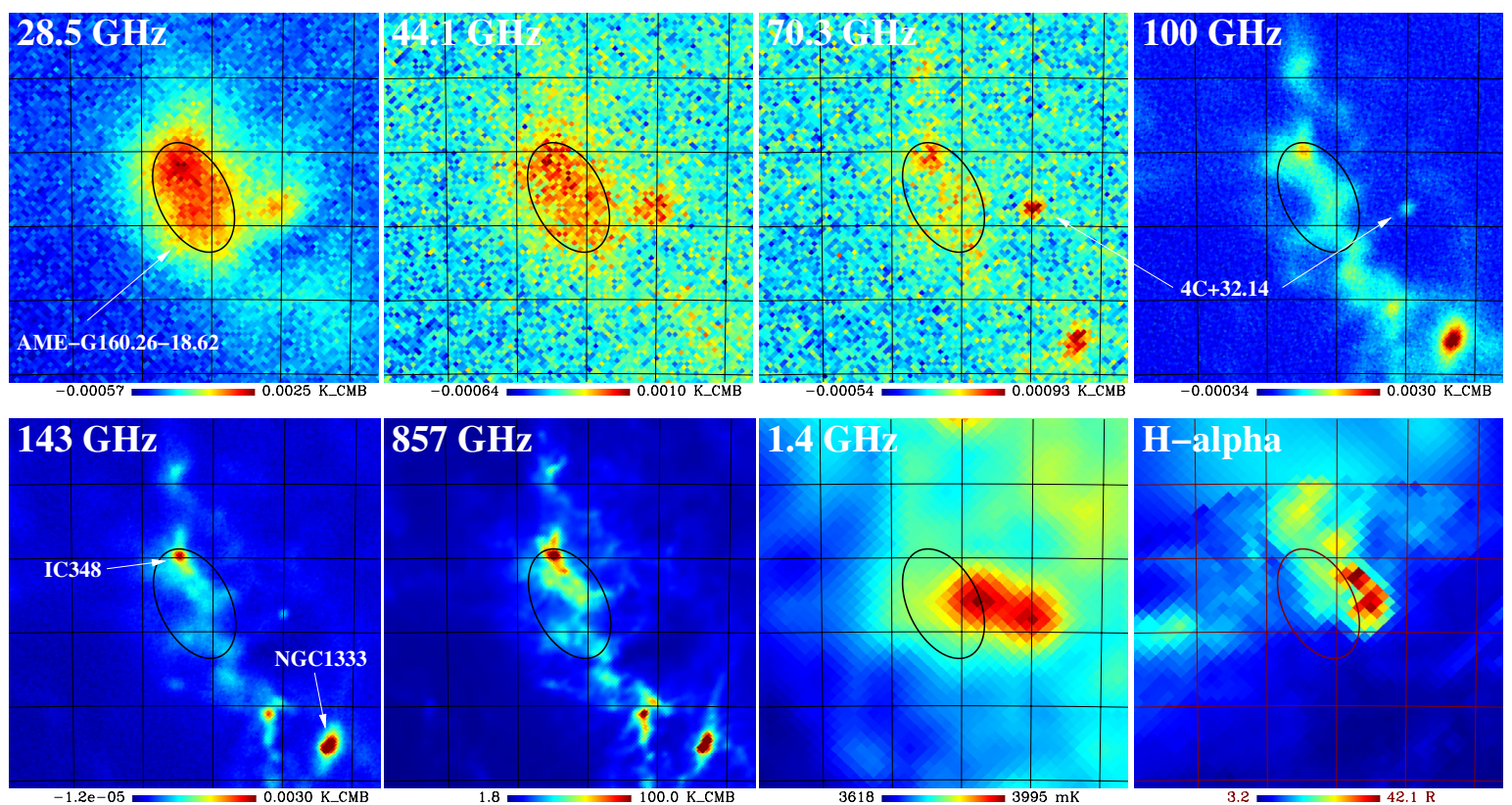

Fig. 1. Maps of the Perseus molecular cloud region at their original angular resolution. From left to right, top row: Planck 28.5; $44.1 ; 70.3$ and $100 \mathrm{GHz}$. Bottom row: Planck 143 and $857 \mathrm{GHz} ; 1.4 \mathrm{GHz}$; and $\mathrm{H} \alpha$. The maps cover $5^{\circ} \times 5^{\circ}$ centred on $(l, b)=\left(160^{\circ} .26,-18^{\circ} .62\right)$ and have linear colour scales. The graticule has $1^{\circ}$ spacing in Galactic coordinates. The FWHM of the elliptical Gaussian model used to fit the flux density in the filtered maps (see text) is shown. The strong AME is evident at 30-70 GHz.

diffuse emission at angular scales $\gtrsim 1^{\circ}$ and this will be described in Sect. 4. A $10 \%$ overall calibration uncertainty is assumed.

\subsection{WMAP data}

WMAP 7-year data are included in the analysis (Jarosik et al. 2011). The data span 23-94 GHz and thus complement Planck data, particularly the $K$-band $(22.8 \mathrm{GHz})$ channel. We use the $1^{\circ}$ smoothed maps available from the LAMBDA website. We apply colour corrections to the central frequencies using the recipe described by Jarosik et al. (2003) and assume a conservative 3\% overall calibration uncertainty.

\subsection{DIRBE data}

To sample the peak of the blackbody curve for temperatures $\gtrsim 15 \mathrm{~K}$ we include the COBE-DIRBE data at $240 \mu \mathrm{m}$ (1249 GHz), $140 \mu \mathrm{m}(2141 \mathrm{GHz})$ and $100 \mu \mathrm{m}(2997 \mathrm{GHz})$. The DIRBE data are the Zodi-Subtracted Mission Average (ZSMA) maps (Hauser et al. 1998) regridded into the HEALPix format. Colour corrections were applied as described in the DIRBE explanatory supplement version 2.3. Data at higher frequencies are not considered, since these will be dominated by transiently heated grains which are not in thermal equilibrium with the interstellar radiation field and therefore cannot be modelled with a single modified blackbody curve. Furthermore, at wavelengths $\lesssim 40 \mu \mathrm{m}$ the spectrum is dominated by many emission/absorption lines.

\section{Perseus molecular cloud}

\subsection{Introduction and maps}

The Perseus molecular cloud complex is a relatively nearby giant molecular cloud of $1.3 \times 10^{4} M_{\odot}$ at a distance of $260 \mathrm{pc}$
(Cernicharo et al. 1985) having an angular extent of $6^{\circ} \times 2^{\circ}$. It contains a chain of six dense cores (B 5, IC 348, B 1, NGC 1333, L 1455 and L 1448) with $A_{v}>2$ mag running northeastsouthwest and with two main centres of medium-mass star formation associated with the reflection nebulae IC 348 and NGC 1333 near each end (see Fig. 1). As a nearby example of low-to-medium mass star formation it has been extensively studied, with the Spitzer c2d Legacy Program (Evans et al. 2003) and the COMPLETE survey (Ridge et al. 2003), which provide a wealth of infrared and spectral line data to understand conditions throughout the cloud.

Anomalous emission was first detected as an excess in this region of the sky via the Tenerife experiment (Davies et al. 1987) at $5^{\circ}$ angular scales, and it contributed most of the dust-correlated signal found by de Oliveira-Costa et al. (1999). With the $1^{\circ}$ beam and $11-17 \mathrm{GHz}$ frequency bands of the COSMOSOMAS experiment (Gallegos et al. 2001) it was possible to locate and identify the Perseus molecular cloud as the strong source of this emission (Watson et al. 2005). Follow-up observations with the VSA interferometer (Tibbs et al. 2010) at $10^{\prime}$ resolution detected five "hot-spots" of anomalous emission, one of which corresponds to the dense region $\left(A_{\mathrm{v}} \gtrsim 10\right)$ around the IC 348 core. The other four hot-spots are associated with nearby dense dust knots that lie on a warm ring of dust emission (G159.6-18.5), about 90' to the south-east of IC 348. This ring, which is seen at $100 \mu \mathrm{m}$, is thought to be warmed by the OB star HD 278942 (de Zeeuw et al. 1999), which is also responsible for a weak $\mathrm{H}$ in region in the centre of the ring, and which can be seen in the low-frequency continuum surveys with an integrated flux density of a few janskys. The AME appears to have little or no polarisation, with an upper limit of $1.8 \%$ at $33 \mathrm{GHz}$ (Lopez-Caraballo et al. 2011).

Planck maps of the Perseus molecular cloud region, covering 30-857 GHz, are shown in Fig. 1, along with the $1.4 \mathrm{GHz}$ and $\mathrm{H} \alpha$ maps. For display purposes the Planck maps have 

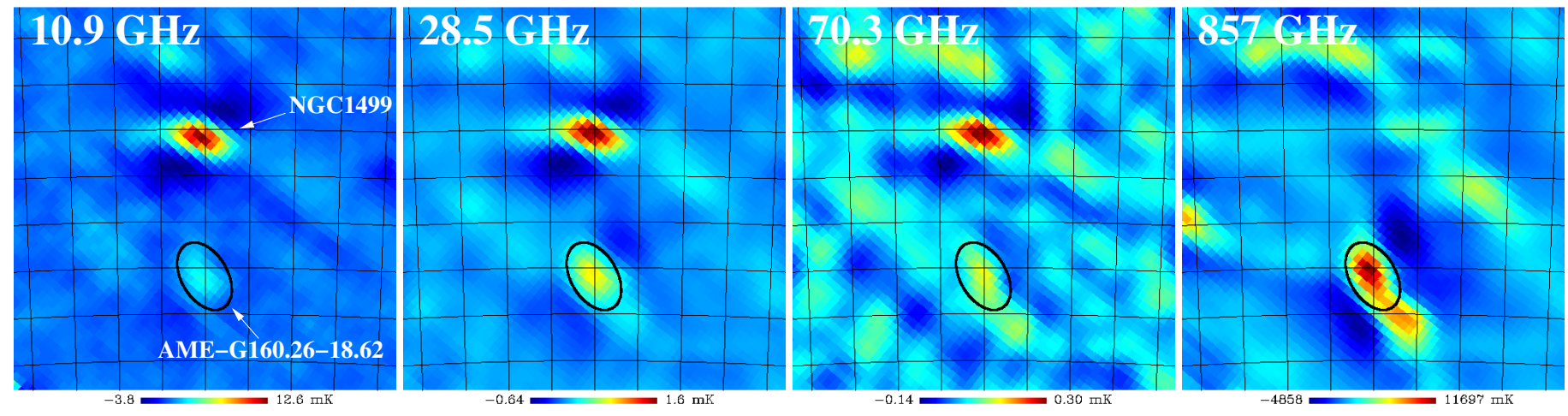

Fig. 2. $16^{\circ} \times 16^{\circ}$ maps after filtering with the COSMOSOMAS data reduction pipeline. The maps are centred at $(l, b)=\left(160^{\circ},-15^{\circ}\right)$ and the graticule spacing is $2^{\circ}$ in Galactic coordinates. From left to right: COSMOSOMAS $10.9 \mathrm{GHz}$; Planck $28.5 \mathrm{GHz} ; 70.3 \mathrm{GHz}$; and $857 \mathrm{GHz}$. The elliptical Gaussian model used to calculate the spectrum is indicated.

been CMB-subtracted as described in Zacchei et al. (2011) and Planck HFI Core Team (2011b) and the units are thermodynamic kelvins. The strong dust-correlated AME at $30-70 \mathrm{GHz}$ is evident; it has no obvious counterpart at $1.4 \mathrm{GHz}$ but correlates well with the higher $(>100 \mathrm{GHz})$ Planck frequencies, which are dominated by thermal dust. The majority of the AME comes from the northeast end of the molecular cloud, which harbours the dense IC 348 reflection nebula. Some weak free-free emission is seen to the east and the contribution from the extragalactic radio source $4 \mathrm{C}+32.14$ is clear and must be removed (Sect. 4.2). The $\mathrm{H} \alpha$ (and $1.4 \mathrm{GHz}$ emission) morphology is not co-located with the emission seen at $30-70 \mathrm{GHz}$, but is likely to be significantly absorbed by the intervening dust. Nevertheless, the brightest $\mathrm{H} \alpha$ pixels predict $\approx 0.25 \mathrm{mK}$ at $30 \mathrm{GHz}$ (Dickinson et al. 2003), a factor of ten below the AME at $30 \mathrm{GHz}$. It is interesting that there is considerable bright structure observed in the Planck HFI maps, which is not always associated with strong AME. This is likely to be a consequence of a different environment and dust grain population.

\subsection{Spectrum}

To reduce correlated $1 / f$ noise from the atmosphere/receiver the COSMOSOMAS time-ordered data have been filtered with the suppression of the first seven harmonics in the FFT of the circular scans. To compare with flux densities from other instruments one must either correct for the flux lost in filtering or filter all the other data in the same way. With extended sources such as Perseus there is uncertainty in estimating a single correction factor to take into account the filtering process, which in turn directly affects the shape of the frequency spectrum. Filtering all the data in the same way retains the spectral shape and ensures that the spatial frequencies used to form the spectrum are common between the different data sets.

The first step is to smooth all the data to the same resolution as the lowest frequency channel of the COSMOSOMAS data, i.e., a FWHM of 1․12. Each data set is then filtered using the COSMOSOMAS reduction pipeline and binned into a map as was done with the original COSMOSOMAS data (Fernández-Cerezo et al. 2006; Hildebrandt et al. 2007). Example maps at 10.9, 28.5, 70.3, and $857 \mathrm{GHz}$ are shown in Fig. 2. To estimate the flux density at each frequency the filtered maps were compared to a filtered model map, which was passed through the same scan strategy. For the model we use an elliptical Gaussian $\left(1.6 \times 1^{\circ} .0\right.$ with major-axis PA $\left.51^{\circ}\right)$, centred at $(\mathrm{RA}, \mathrm{Dec})=\left(55^{\circ} .33,+31^{\circ} .67\right)$ or $(l, b)=\left(160^{\circ} .26,-18.62\right)$, following Watson et al. (2005); this gives a solid angle $\Omega=$ $5.52 \times 10^{-4} \mathrm{sr}$. We will refer to this as AME-G160.26-18.62. There is a flat-spectrum radio quasar, 4C+32.14 (NRAO 140), within $1^{\circ}$, with a flux density of $\approx 1-3 \mathrm{Jy}$, which is seen in the Planck Early Release Compact Source Catalogue (ERCSC) at frequencies up to $143 \mathrm{GHz}$ (Planck Collaboration 2011c). We use the flux densities and beam widths taken from the ERCSC and subtract the two-dimensional Gaussian profile from the maps before smoothing and filtering. Meanwhile in the COSMOSOMAS maps we must extrapolate the flux density of $4 \mathrm{C}+32.14$ to the appropriate frequencies and simulate its contribution to the filtered map; we assume $2.0 \mathrm{Jy}$ at $11-19 \mathrm{GHz}$, which is in good agreement with NVSS (Condon et al. 1998) and recent $2.7 \mathrm{GHz}$ measurements made with the Effelsberg telescope (Reich \& Reich 2009). The contribution of $4 \mathrm{C}+32.14$ is $\approx 5 \%$ of the integrated flux density, so the final results are not very sensitive to the exact treatment of the subtraction or the variability of the source. Both the smoothing and filtering result in correlated noise between the pixels in the map. A correlation analysis (e.g., Davies et al. 2006) is used to calculate the flux density taking into account the correlated noise. The covariance matrix is estimated from the size of the smoothing kernel and the point source response to the scan strategy. A simulated map is produced for the source, with an integrated flux density of $1 \mathrm{Jy}$, and a maximum likelihood method is used to find the amplitude and error for the given frequency channel data within a $19 \times 19$ pixel box $(6.3 \times 6.3)$ around the source position.

As a control, we tested the simulation and filtering pipeline by applying the technique to the extended California nebula $\mathrm{H}_{\text {II }}$ region (NGC 1499), approximately $6^{\circ}$ to the north of Perseus at $(l, b)=\left(160^{\circ} 60,-12^{\circ} .05\right)$, as seen in Fig. 2 . The emission at radio/microwave wavelengths is dominated by optically thin free-free emission. Flux densities at frequencies $>100 \mathrm{GHz}$ were affected by dust emission lying adjacent to the north-west of NGC 1499 and thus were not included. The spectrum is shown in Fig. 3. A simple power-law fit, with a best-fitting spectral index $\alpha=-0.05 \pm 0.01$, is overplotted. The fit is relatively $\operatorname{good}\left(\chi^{2} /\right.$ d.o.f. $\left.=1.23\right)$ but there is some evidence for AME at frequencies $\approx 30-100 \mathrm{GHz}$. Furthermore, the spectral index is slightly flatter than the value expected for optically thin freefree emission over this frequency range, $\alpha \approx-0.1$ to -0.15 (Dickinson et al. 2003). Indeed, AME has been observed to be 


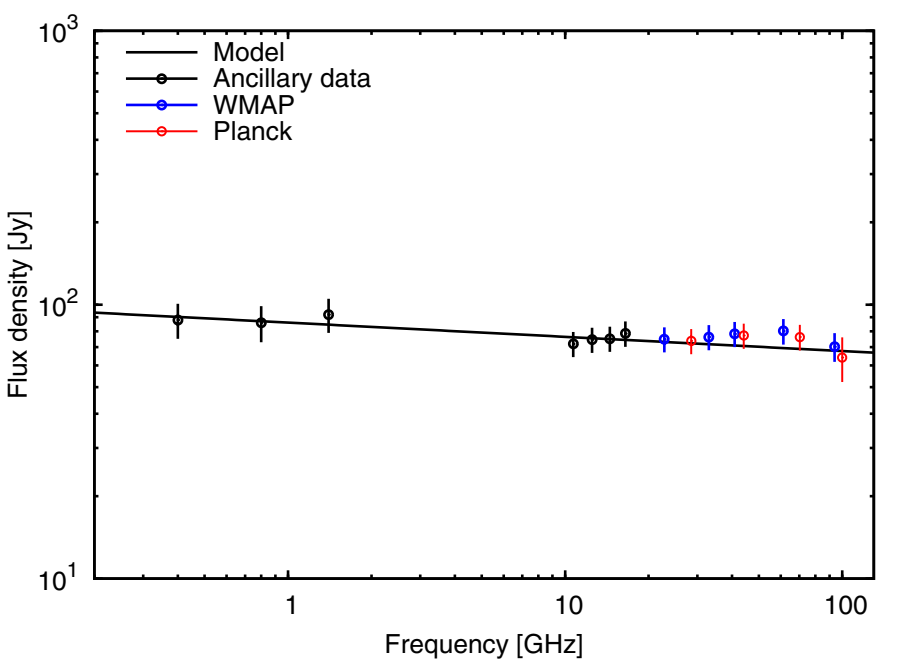

Fig. 3. Spectrum of the California nebula (NGC 1499), measured using the filtered flux method (see text). A simple power-law fit is shown.

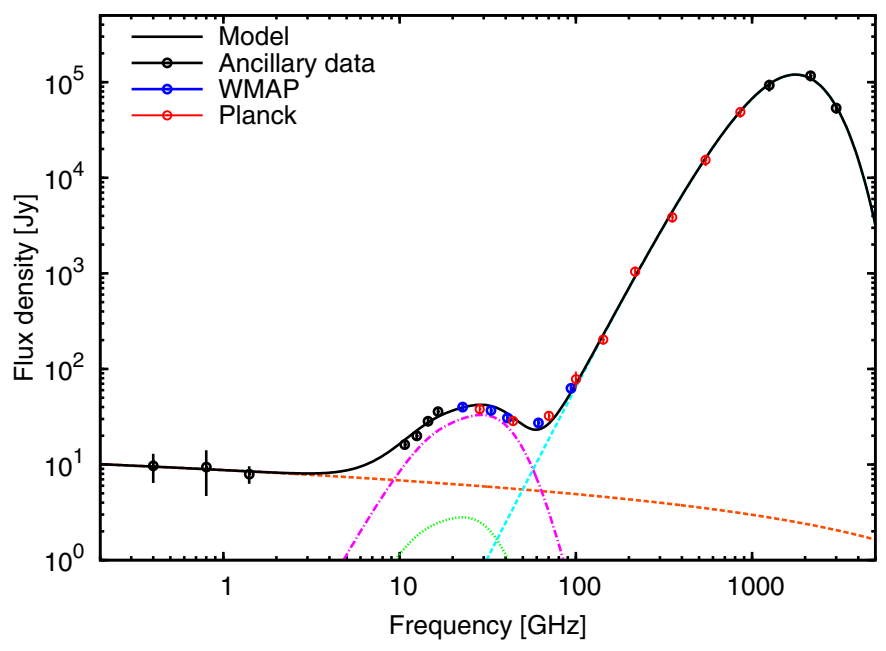

Fig. 4. Spectrum of AME-G160.26-18.62 in the Perseus molecular cloud. The best-fitting model consisting of free-free (orange dashed line), spinning dust, and thermal dust (light blue dashed line) is shown. The two-component spinning dust model consists of high density molecular gas (magenta dot-dashed line) and low density atomic gas (green dotted line).

significant in some H II regions (Dickinson et al. 2009; Todorović et al. 2010). We confirmed the spectral shape with a standard aperture photometry analysis (as described in Sect. 5) and will investigate this in more detail in a future publication. Note that the excess at $30-100 \mathrm{GHz}$ disappears when the spectrum is calculated away from the nearby dust feature to the north-west of NGC 1499. Given the consistency of the flux densities between the frequencies, and that we have obtained similar results using standard aperture photometry, we are confident that the spectrum calculated using the filtered maps is robust.

The spectrum for AME-G160.26-18.62 in Perseus is shown in Fig. 4. The flux densities and associated errors are listed in Table 2. The spectrum is well sampled across the radio, microwave and FIR regimes and is a significant improvement on that presented in Watson et al. (2005), with the additional Planck data allowing a much more accurate spectrum of the AME to be extracted. The low-frequency $(<2 \mathrm{GHz})$ data show a flat spectrum consistent with free-free emission while the high
Table 2. Flux densities for AME-G160.26-18.62 in the Perseus molecular cloud and residual fluxes when free-free, $\mathrm{CMB}$ and thermal dust components are removed.

\begin{tabular}{lcc}
\hline \hline $\begin{array}{l}\text { Frequency } \\
{[\mathrm{GHz}]}\end{array}$ & $\begin{array}{c}\text { Flux density } \\
{[\mathrm{Jy}]}\end{array}$ & $\begin{array}{c}\text { Flux density residual } \\
{[\mathrm{Jy}]}\end{array}$ \\
\hline 0.408 & $9.7 \pm 3.3$ & $0.2 \pm 3.4$ \\
0.82 & $9.4 \pm 4.7$ & $0.5 \pm 4.8$ \\
1.42 & $8.0 \pm 1.7$ & $-0.5 \pm 1.8$ \\
10.9 & $16.1 \pm 1.8$ & $9.4 \pm 2.0$ \\
12.7 & $20.0 \pm 2.2$ & $13.3 \pm 2.4$ \\
14.7 & $28.4 \pm 3.1$ & $21.8 \pm 3.3$ \\
16.3 & $35.8 \pm 4.0$ & $29.3 \pm 4.2$ \\
22.8 & $39.8 \pm 4.2$ & $33.4 \pm 4.5$ \\
28.5 & $38.1 \pm 4.0$ & $31.5 \pm 4.4$ \\
33.0 & $36.7 \pm 3.9$ & $29.7 \pm 4.4$ \\
40.9 & $30.8 \pm 3.3$ & $22.6 \pm 4.0$ \\
44.1 & $28.5 \pm 3.2$ & $19.6 \pm 4.0$ \\
61.3 & $27.4 \pm 3.4$ & $10.9 \pm 6.4$ \\
70.3 & $32.2 \pm 3.9$ & $8.9 \pm 9.0$ \\
93.8 & $63.0 \pm 7.8$ & $7 \pm 21$ \\
100 & $78 \pm 15$ & $10 \pm 29$ \\
143 & $202 \pm 22$ & $-25 \pm 80$ \\
217 & $1050 \pm 130$ & $120 \pm 320$ \\
353 & $3860 \pm 470$ & $-600 \pm 1400$ \\
545 & $15300 \pm 2100$ & $-800 \pm 4900$ \\
857 & $48700 \pm 6100$ & $-1000 \pm 14000$ \\
1250 & $93000 \pm 13000$ & $-2400 \pm 28000$ \\
2143 & $117000 \pm 15000$ & $7000 \pm 35000$ \\
2997 & $53600 \pm 6700$ & $-2000 \pm 20000$ \\
\hline
\end{tabular}

Notes. The fitted spinning dust model consists of two components.

frequencies $(>100 \mathrm{GHz})$ are dominated by thermal dust emission. The excess at $\approx 10-70 \mathrm{GHz}$ is evident and has a peaked (convex) spectrum, with a maximum at $25 \mathrm{GHz}$.

The data allow us to fit a multi-component parametric model to the flux density spectrum. The model consists of four components: free-free emission; thermal dust emission; a CMB fluctuation; and spinning dust emission. The sum is

$S=S_{\mathrm{ff}}+S_{\mathrm{td}}+S_{\mathrm{cmb}}+S_{\mathrm{sp}}$.

The free-free flux density, $S_{\mathrm{ff}}$, is calculated from the brightness temperature, $T_{\mathrm{ff}}$, based on the optical depth, $\tau_{\mathrm{ff}}$, using the standard formulae:

$S_{\mathrm{ff}}=\frac{2 k T_{\mathrm{ff}} \Omega v^{2}}{c^{2}}$,

where $k$ is the Boltzmann constant, $\Omega$ is the solid angle, and $v$ is the frequency,

$T_{\mathrm{ff}}=T_{\mathrm{e}}\left(1-\mathrm{e}^{-\tau_{\mathrm{ff}}}\right)$,

and the optical depth, $\tau_{\mathrm{ff}}$, is given by

$\tau_{\mathrm{ff}}=3.014 \times 10^{-2} T_{\mathrm{e}}^{-1.5} v^{-2} E M g_{\mathrm{ff}}$,

where $T_{\mathrm{e}}$ is the electron temperature (in units of $\mathrm{K}$ ), $E M$ is the emission measure (in units of $\mathrm{cm}^{-6} \mathrm{pc}$ ) and $g_{\mathrm{ff}}$ is the Gaunt factor, which is approximated as

$g_{\mathrm{ff}}=\ln \left(\frac{4.955 \times 10^{-2}}{v / \mathrm{GHz}}\right)+1.5 \ln \left(T_{\mathrm{e}}\right)$.

It is the Gaunt factor that results in a slight steepening of the freefree spectrum with frequency, which is particularly pronounced at $\gtrsim 100 \mathrm{GHz}$. 
The thermal dust is modelled as a single-component, modified blackbody curve, $v^{\beta} B\left(v, T_{\mathrm{d}}\right)$, which we normalise using an optical depth at $250 \mu \mathrm{m}(1.2 \mathrm{THz}), \tau_{250}$, as

$S_{\mathrm{td}}=2 h \frac{v^{3}}{c^{2}} \frac{1}{\mathrm{e}^{h v / k T_{\mathrm{d}}}-1} \tau_{250}(v / 1.2 \mathrm{THz})^{\beta} \Omega$

where $h$ is the Planck constant and $\beta$ is the emissivity index. The $\mathrm{CMB}$ anisotropy is evaluated as the differential of the blackbody temperature function, in thermodynamic units $\left(\Delta T_{\mathrm{CMB}}\right)$, converted to flux density units,

$S_{\mathrm{cmb}}=\left(\frac{2 k \Omega v^{2}}{c^{2}}\right) \Delta T_{\mathrm{CMB}}$

The spinning dust model is based on an assumed theoretical emissivity curve, $j_{v}$ (in units of $\mathrm{Jy} \mathrm{sr}^{-1} \mathrm{~cm}^{2} \mathrm{H}^{-1}$ ), normalised by the average column density per $\mathrm{H}$ nucleon, $N_{\mathrm{H}}$, and solid angle, $\Omega$,

$S_{\mathrm{sp}}=N_{\mathrm{H}} j_{v} \Omega$.

As described in Sect. 6, we use the SPDUST code (Ali-Haïmoud et al. 2009) to model the spinning dust spectrum.

The best-fitting model is plotted in Fig. 4. The fitted parameters are $E M$ for free-free, differential temperature of $\mathrm{CMB}$ perturbation $\left(\Delta T_{\mathrm{CMB}}\right)$, dust temperature $\left(T_{\mathrm{d}}\right)$, dust emissivity index $(\beta)$ and $250 \mu \mathrm{m}$ opacity $\left(\tau_{250}\right)$. The spinning dust model consists of two components, to represent dust associated with dense molecular gas and low density atomic gas, and will be discussed in Sect. 6. The electron temperature is assumed to have a typical value of $8000 \mathrm{~K}$ for gas in the solar neighbourhood. A conservative $3 \%$ uncertainty in the $\mathrm{CO}$ correction is assumed at $100 \mathrm{GHz}$ and is not included in the fit. Similarly, the $217 \mathrm{GHz}$ value is not included in the fit, due to the possible small contamination from the $\mathrm{CO} J=2 \rightarrow 1$ line. From the initial spectral fit the spectral index is found at each frequency, which is then used to calculate the appropriate colour corrections for each band in an iterative manner. After applying the colour corrections (typically a few per cent correction), the spectrum is fitted again to obtain the final flux densities, which are given in Table 2. The uncertainty in the fitted model for the spectrum is computed by propagating the errors of each of the parameters and combining them with the other sources of error, i.e., background/noise residuals and an overall calibration error. The fit is very good with $\chi^{2} /$ d.o.f. $=0.95$. The best-fitting parameters are: $E M=148 \pm 13.5 \mathrm{~cm}^{-6} \mathrm{pc}$ for free-free; $T_{\mathrm{d}}=18.5 \pm 0.6 \mathrm{~K}$ and $\beta=1.65 \pm 0.08$ for the thermal dust; and a negligible CMB contribution of $\Delta T_{\mathrm{CMB}}=-6 \pm 66 \mu \mathrm{K}$.

In Fig. 5 we show the residual spectrum after subtracting the free-free, $\mathrm{CMB}$ and thermal dust models. The additional uncertainty from the subtraction of the models is added in quadrature to the flux density errors to find the final uncertainties on the residual flux densities, which are listed in Table 2. This represents the most precise spectrum of AME to date. The weighted average over the range 10 to $94 \mathrm{GHz}$ gives a detection significance level of $17.1 \sigma$. The peak of the spinning dust component is centred at $\approx 25 \mathrm{GHz}$ and the low and high frequency sides are well-defined in the range 10-90 GHz. A physical model for the spinning dust is presented in Sect. 6. It consists of two components (atomic and molecular), which are overplotted in Fig. 5, and is seen to be an excellent fit to the data.

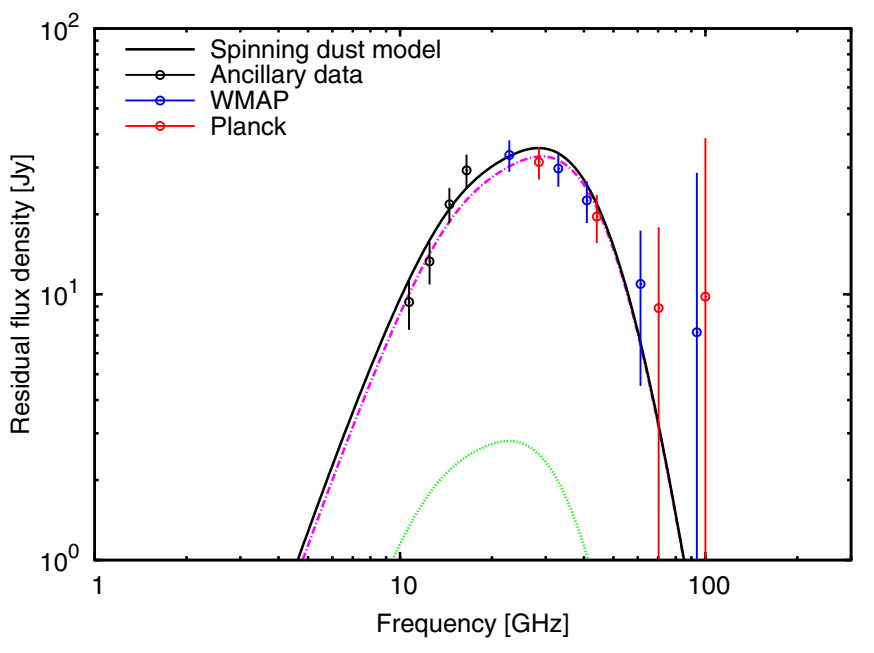

Fig. 5. Spectrum of AME-G160.26-18.62 in the Perseus molecular cloud after subtracting the best-fit free-free, CMB and thermal dust components. A theoretical spinning dust model, consisting of two components is shown as the black solid line; the magenta dot-dashed line is for high density molecular gas and the green dotted line is for low density atomic gas, which is a very small contribution in this case (see Sect. 6).

\section{5. $\rho$ Ophiuchi molecular cloud}

\subsection{Introduction and maps}

The $\rho$ Ophiuchi molecular cloud (e.g., Encrenaz 1974; Kulesa et al. 2005; Young et al. 2006) lies in the Gould Belt of the closest molecular complexes, at a distance $D=135 \pm 15 \mathrm{pc}$ (Perryman et al. 1997). It is undergoing intermediate-mass star formation. Ultra-violet radiation from its hottest young stars heats and dissociates exposed layers, but does not ionise bulk hydrogen. The most prominent PDR in $\rho$ Ophiuchi is the $\rho$ Oph W filament, with $n_{\mathrm{H}}=10^{4}-10^{5} \mathrm{~cm}^{-3}$, which has been studied by Liseau et al. (1999) and Habart et al. (2003). $\rho$ Oph W is excited by HD 147889 (a B2, 3 IV binary), the earliest star in the $\rho$ Oph star formation complex. The line of sight to HD 147889 can be taken to characterise the physical conditions in the ambient diffuse cloud, with $N_{\mathrm{H}} \sim 2 \times 10^{20} \mathrm{~cm}^{-2}, n_{\mathrm{H}} \sim 400 \mathrm{~cm}^{-3}$; no accurate values are available for $N_{\mathrm{H}_{2}}$ (van Dishoeck \& Black 1989; Kaźmierczak et al. 2010). The bulk of the mass in the $\rho$ Ophiuchi cloud is situated in the Oph A molecular core (e.g., Wootten et al. $1978,1979)$, located roughly $10^{\prime}$ southwest of $\rho$ Oph W.

Only faint radiation from the Rayleigh-Jeans tail of $\sim 10-100 \mathrm{~K}$ dust is expected at wavelengths longwards of $\sim 3 \mathrm{~mm}$. Yet CBI observations revealed that the $\rho$ Oph W PDR is surprisingly bright at centimetre wavelengths (Casassus et al. 2008). The WMAP $33 \mathrm{GHz}$ image confirms that the general location of the signal seen by CBI is offset by $10^{\prime}$ from the centroid of the WMAP $94 \mathrm{GHz}$ data, located on the Oph A core. The signal seen at centimetre wavelengths has no $5 \mathrm{GHz}$ counterpart; instead the optically thin free-free emission is located in the immediate vicinity of HD 147889. The other early-type stars in the complex are S 1 (B4 V) and SR 3 (B6 V), and each is surrounded by bright IR emission peaks, which lack any radio counterpart.

Planck maps of the $\rho$ Ophiuchi molecular cloud region covering $30-857 \mathrm{GHz}$, along with the $1.4 \mathrm{GHz}$ and $\mathrm{H} \alpha$ maps, are shown in Fig. 6. Strong AME is evident at 28.5-44.1 GHz, with the bulk of the emission falling within 0.5 of the central position. For display purposes the Planck maps have been CMBsubtracted as described in Zacchei et al. (2011) and Planck HFI 

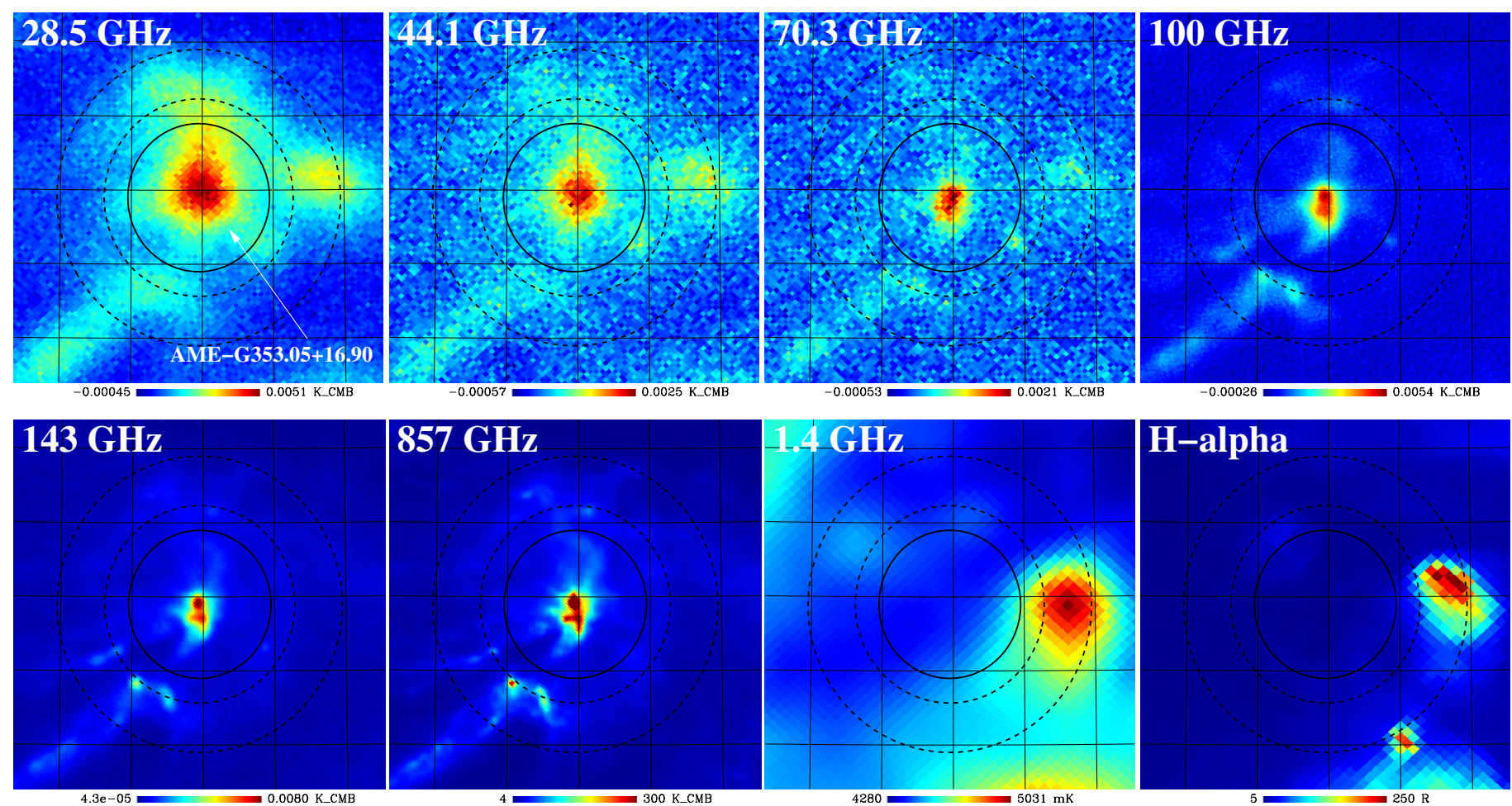

Fig. 6. Maps of the $\rho$ Ophiuchi molecular cloud region at their original angular resolution. From left to right, top row: Planck 28.5; 44.1; 70.3; and $100 \mathrm{GHz}$. Bottom row: Planck 143 and $857 \mathrm{GHz} ; 1.4 \mathrm{GHz}$; and $\mathrm{H} \alpha$. The maps cover $5^{\circ} \times 5^{\circ}$ centred on $(l, b)=\left(353^{\circ} .05,+16^{\circ} .90\right)$ and have linear colour scales. The graticule has $1^{\circ}$ spacing in Galactic coordinates. The circular aperture and background annulus, which were used to calculate the flux density (see text), are indicated. The strong AME is evident at 28.5 and $44.1 \mathrm{GHz}$.

Core Team (2011b) and the units are thermodynamic kelvins. The $\mathrm{H}_{\text {II }}$ region to the west is also clearly detected in the Planck LFI maps, but is well separated from the bulk of the AME. In the location of the strongest AME, there is a valley in intensity seen at low frequencies, supported by little $\mathrm{H} \alpha$ emission. The AME is well correlated with Planck HFI maps, indicating an association with dust.

\subsection{Spectrum}

The $\rho$ Ophiuchi molecular cloud (at declination $-24^{\circ}$ ) lies outside the range of the COSMOSOMAS survey. We therefore chose to use simple aperture photometry on the unfiltered maps to calculate the spectrum. The maps were first smoothed to a common resolution of $1^{\circ}$ and converted to units of Jy pixel ${ }^{-1}$. The brightness in each pixel was then summed over a given aperture to give the flux density. We chose a circular aperture centred at $(l, b)=\left(353^{\circ} .05,16^{\circ} .90\right)$ with a radius of $60^{\prime}$; this corresponds to a solid angle $\Omega=9.57 \times 10^{-4}$ sr. We will refer to this source as AME-G353.05+16.90. An estimate of the background brightness is subtracted using the median value in a circular annulus between an inner radius of $80^{\prime}$ and an outer radius of $120^{\prime}$, as indicated in Fig. 6. This removes local background emission and any residual offsets in the maps. Errors are estimated from the standard deviation in the background annulus, which was verified with simulations of random sources of known flux density that were injected into the sky maps. The results were not strongly sensitive to the exact choice of background annulus; all values were within the $1 \sigma$ uncertainties. This indicates that the emission in the aperture is bright relative to the local background fluctuations. We also compared two halves of the data as a jack-knife test. Results were found to be consistent to within a fraction of the derived uncertainties.

We tested the aperture photometry by measuring the spectrum of well-known, bright objects. We chose bright $\mathrm{H}$ in regions, which are expected to be dominated by thermal bremsstrahlung (free-free) radiation at radio frequencies and thermal dust in the sub-mm/FIR. Figure 7 shows the spectrum of the Orion nebula (M42) with an aperture of radius $60^{\prime}$ and background annulus between radii of $80^{\prime}$ and $120^{\prime}$. At frequencies below a few gigahertz, the free-free emission is becoming optically thick, with a turnover frequency of $\approx 2 \mathrm{GHz}$. At frequencies $\sim 10-60 \mathrm{GHz}$, the spectrum is well-fitted by a simple optically thin free-free spectrum $(\alpha \approx-0.1)$. The WMAP and Planck data points are in good agreement to within $\approx 1 \%$; the residuals are consistent with zero and within a fraction of the assumed uncertainties. At frequencies $\gtrsim 100 \mathrm{GHz}$, thermal dust emission dominates and is well described by a modified blackbody with an emissivity index $\beta=1.71 \pm 0.12$ and dust temperature $T_{\mathrm{d}}=25.7 \pm 1.7 \mathrm{~K}$.

We note that the lack of AME in M42 is likely to be due to the difference in conditions (e.g., intense radiation field) that may result in depletion of the smallest dust grains, which would result in a large reduction in the amount of spinning dust emission. This is a different situation to the more diffuse warm ionised medium (WIM) studied by Dobler et al. (2009), where they detected AME in the WIM at the level of $\approx 20 \%$ relative to the free-free emission.

Figure 8 shows the spectrum of AME-G353.03+16.90 in the $\rho$ Oph West region; the flux densities are listed in Table 3. The same model used for the Perseus region (Sect. 4.2) was 


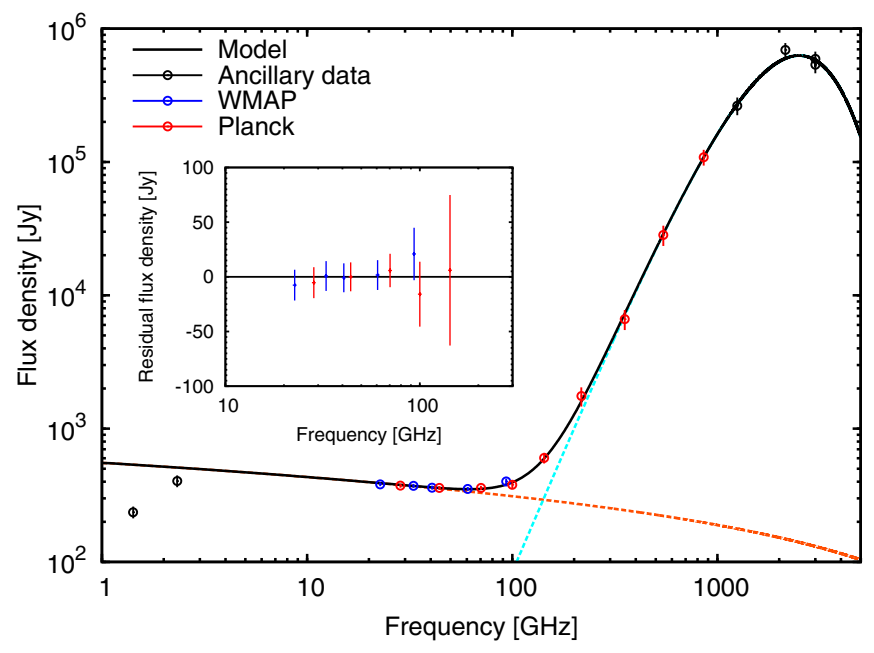

Fig. 7. Spectrum of the Orion nebula (M 42) $\mathrm{H}$ II region using aperture photometry. The model consists of optically thin free-free emission (orange dashed line) and thermal dust emission (light blue dashed line). The inner panel shows the residual spectrum after removal of the model, indicating the consistency between Planck and WMAP data.

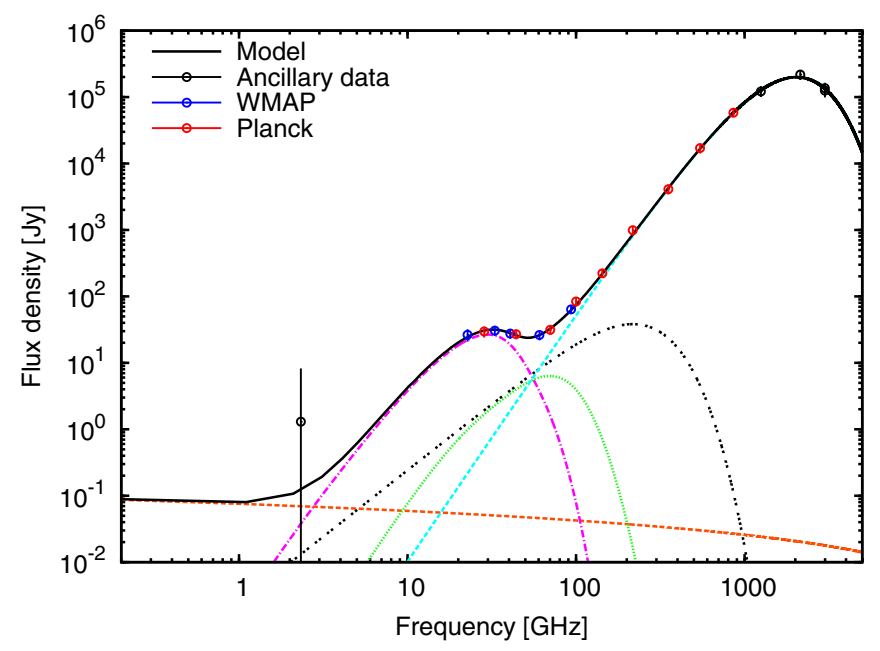

Fig. 8. Spectrum of AME-G353.05+16.90 in the $\rho$ Ophiuchi West molecular cloud. The best-fitting model consisting of free-free (orange dashed line), spinning dust, CMB (black double/triple-dotted line), and thermal dust (light blue dashed line), is shown. The spinning dust model consists of two components: high density molecular gas (magenta dot-dashed line); and low density atomic gas (green dotted line). The 100/217 GHz data are contaminated by CO line emission and are not included in the fit.

fitted to the data, except for the details of the spinning dust component, which will be discussed in Sect. 6. Significant CO line contamination is visible at 100 and $217 \mathrm{GHz}$, so these two bands were not included in the fit. There is minimal free-free emission within the aperture, and hence the error at low (0.4$2.3 \mathrm{GHz}$ ) frequencies is dominated by fluctuations in the local background, as indicated by the large error in the $2.3 \mathrm{GHz}$ flux density. Note that the $408 / 1420 \mathrm{MHz}$ data were included in the fit but were consistent with zero. The best-fitting parameters are: $E M=1 \pm 48 \mathrm{~cm}^{-6} \mathrm{pc} ; T_{\mathrm{d}}=20.7 \pm 1.6 \mathrm{~K} ; \beta=1.75 \pm 0.18$; and $\mathrm{CMB}$ contribution $\Delta T_{\mathrm{CMB}}=82 \pm 61 \mu \mathrm{K}$. The fit of the overall model is very good $\left(\chi^{2} /\right.$ d.o.f. $\left.=0.29\right)$.

As a test of the impact that the Planck data was having on the spectrum, we repeated the analysis omitting the Planck data.
Table 3. Flux densities for AME-G353.05+16.90 in the $\rho$ Ophiuchi molecular cloud and residual flux densities when free-free, CMB and thermal dust components are removed.

\begin{tabular}{lcc}
\hline \hline $\begin{array}{l}\text { Frequency } \\
{[\mathrm{GHz}]}\end{array}$ & $\begin{array}{c}\text { Flux density } \\
{[\mathrm{Jy}]}\end{array}$ & $\begin{array}{c}\text { Flux density residual } \\
{[\mathrm{Jy}]}\end{array}$ \\
\hline 0.4 & $-6.8 \pm 9.4$ & $-7 \pm 11$ \\
1.4 & $-1.0 \pm 6.8$ & $-1.0 \pm 8.3$ \\
2.3 & $1.3 \pm 6.9$ & $1.2 \pm 8.3$ \\
22.7 & $26.3 \pm 5.5$ & $24.8 \pm 6.6$ \\
28.5 & $29.6 \pm 5.6$ & $27.3 \pm 6.6$ \\
33.0 & $30.7 \pm 5.3$ & $27.2 \pm 6.3$ \\
40.7 & $27.7 \pm 4.6$ & $21.9 \pm 5.8$ \\
44.1 & $27.0 \pm 4.4$ & $19.9 \pm 5.7$ \\
60.6 & $26.3 \pm 4.5$ & $9.8 \pm 6.5$ \\
70.3 & $31.4 \pm 5.1$ & $6.5 \pm 7.8$ \\
93.4 & $63.6 \pm 8.9$ & $6 \pm 15$ \\
100 & $84 \pm 13$ & $13 \pm 19$ \\
143 & $222 \pm 31$ & $5 \pm 49$ \\
217 & $989 \pm 16$ & $130 \pm 200$ \\
353 & $4100 \pm 670$ & $-180 \pm 780$ \\
545 & $17100 \pm 2900$ & $400 \pm 3200$ \\
857 & $58300 \pm 8800$ & $500 \pm 10000$ \\
1249 & $122000 \pm 22000$ & $-5000 \pm 25000$ \\
2141 & $218000 \pm 37000$ & $20000 \pm 41000$ \\
2997 & $125000 \pm 26000$ & $-9000 \pm 32000$ \\
\hline
\end{tabular}

As expected, the impact was considerable. In particular, the thermal dust model is less well-constrained when the HFI data are not included. For example, the uncertainty in the dust temperature increases to $\pm 2.5 \mathrm{~K}$, while the uncertainty in the emissivity index increases to \pm 0.36 . This is crucial for making precise measurements of the AME at frequencies, particularly above $50 \mathrm{GHz}$ where the subtraction of thermal dust is critical.

In Fig. 9 we show the residual spectrum after subtracting the free-free, $\mathrm{CMB}$, and thermal dust models. The additional error from the subtraction of the models is added in quadrature to the flux density errors to obtain the uncertainty on the residual flux densities, listed in Table 3. As in Perseus, the residual spectrum has a clearly defined convex shape, peaking at a frequency of $30 \mathrm{GHz}$; the excess is significant at the $8.4 \sigma$ level. The spectrum at $50-100 \mathrm{GHz}$ is flatter than a single spinning dust model can easily account for, suggesting a different environment or distribution of dust grains. This may also be due to variations in the dust emissivity index attributable to multiple dust components (e.g., Finkbeiner et al. 1999). To test this possibility would require the inclusion of the $100 / 217 \mathrm{GHz}$ data after careful subtraction of the $\mathrm{CO}$ line contamination.

A theoretical spinning dust model consisting of two components (associated with atomic and molecular gas) is overplotted and provides an excellent fit to the data. Note that the denser molecular gas represents the dominant AME with peak at $\approx 30 \mathrm{GHz}$, while the irradiated low density atomic gas accounts for the $50-100 \mathrm{GHz}$ part of the spectrum. The physical model is presented in Sect. 6.

\section{Modelling the anomalous microwave emission with spinning dust}

Comparisons of the AME with dust emission in localised regions (Casassus et al. 2006; Scaife et al. 2009; Tibbs et al. 2010; Castellanos et al. 2011) and in all-sky surveys (Lagache 2003; Ysard et al. 2010) suggest small dust grains (polycyclic aromatic hydrocarbons, PAH) as carriers of this emission, 


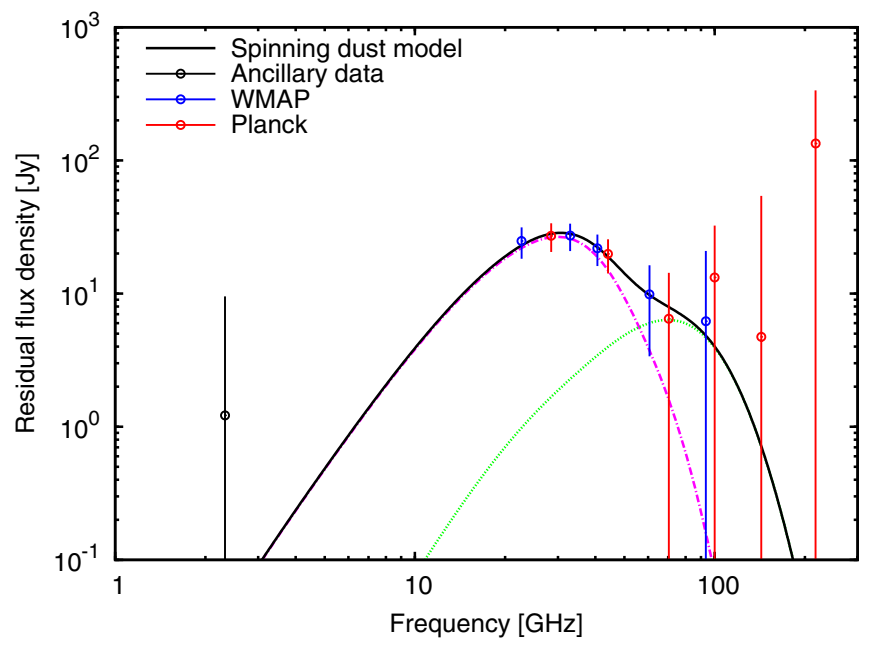

Fig. 9. Spectrum of AME-G353.05+16.90 in the $\rho$ Ophiuchi molecular cloud after subtracting the best-fit free-free, CMB and thermal dust components. A theoretical spinning dust model consisting of two components is shown as the black solid line; the magenta dot-dashed line is for dense molecular gas and the green dotted line is for irradiated low density atomic gas (see Sect. 6). The 100/217 GHz data are contaminated by $\mathrm{CO}$ line emission and are not included in the fit.

supporting the spinning dust explanation (Draine \& Lazarian 1998). The present and previous measurements of the AME show that this component peaks at $20-40 \mathrm{GHz}$ with a brightness of 0.01 to $0.1 \mathrm{MJy} \mathrm{sr}^{-1}$ (see references in Sect. 1). Current spinning dust models (Ali-Haïmoud et al. 2009; Ysard \& Verstraete 2010; Hoang et al. 2010; Silsbee et al. 2011) indicate that lowdensity $\left(n_{\mathrm{H}} \leq 30 \mathrm{~cm}^{-3}\right)$, atomic diffuse gas peaks in this frequency range. Therefore the AME has often been explained with spinning dust emission arising mostly from regions of low density gas, such as from the Cold Neutral Medium (CNM) or Warm Neutral Medium (WNM). The column density $N_{\mathrm{H}}$ associated with the AME can be derived from observations provided the emissivity of spinning dust is known: adopting an average of $10^{-23} \mathrm{MJy} \mathrm{sr}^{-1} \mathrm{~cm}^{2} \mathrm{H}^{-1}$ from theoretical models, we find that $N_{\mathrm{H}} \sim 10^{22} \mathrm{~cm}^{-2}$ for a flux density level of $0.1 \mathrm{MJy} \mathrm{sr}^{-1}$. For a gas density of $30 \mathrm{~cm}^{-3}$, the extent of the emitting region is thus $N_{\mathrm{H}} / n_{\mathrm{H}} \sim 100 \mathrm{pc}$, much larger than the observed size of the present AME clouds $(<10 \mathrm{pc})$. The situation is even worse for the warm ionised medium (WIM) component $\left(n_{\mathrm{H}}<1 \mathrm{~cm}^{-3}\right)$. The spinning dust emission of these regions must therefore include a significant contribution from gas which is denser by at least a factor of 10, as suggested by Casassus et al. (2008) and Castellanos et al. (2011). In the preliminary models of the Perseus and $\rho$ Ophiuchi regions presented below, we show that, assuming plausible physical conditions and PAH size distributions, spinning dust emission from dense gas can explain most of the AME.

The rotation of PAHs in space is governed by IR emission and gas-grain interactions (Draine \& Lazarian 1998). Quantitative modelling of spinning dust emission requires the knowledge of many $(\sim 10)$ parameters describing the state of the gas, the radiation field, and the PAH size distribution, as well as the electric dipole moment. As shown by Ali-Haïmoud et al. (2009) and Ysard et al. (2010), the peak frequency of spinning dust emission in neutral gas (and for radiation intensity $G_{0}<10^{2}$, where $G_{0}$ is the multiplying factor relative to the mean radiation intensity in the interstellar medium, as defined by Mathis et al. 1983) depends chiefly on the gas density and on
Table 4. Parameters in the spinning dust models for Perseus and $\rho$ Ophiuchi.

\begin{tabular}{|c|c|c|c|}
\hline Gas state & Molecular & Atomic & Ionised \\
\hline & & Perseus & \\
\hline$N_{\mathrm{H}}\left[10^{21} \mathrm{~cm}^{-2}\right]$ & 11.7 & 1.3 & 0.4 \\
\hline$n_{\mathrm{H}}\left[\mathrm{cm}^{-3}\right]$ & 250 & 30 & 1 \\
\hline$z[\mathrm{pc}]$ & 15.1 & 14.0 & $\ldots$ \\
\hline$G_{0}$ & 1 & 2 & $\ldots$ \\
\hline$T[\mathrm{~K}]$ & 40 & 100 & $8 \times 10^{3}$ \\
\hline$x_{\mathrm{H}}[\mathrm{ppm}]$ & 112 & 410 & $10^{6}$ \\
\hline$x_{\mathrm{C}}[\mathrm{ppm}]$ & $<1$ & 100 & $\ldots$ \\
\hline$y$ & 1 & 0.1 & $\ldots$ \\
\hline$a_{0}[\mathrm{~nm}]$ & 0.58 & 0.53 & $\ldots$ \\
\hline$b_{\mathrm{C}}[\mathrm{ppm}]$ & 68 & 68 & $\ldots$ \\
\hline$\beta$ & $\ldots$ & 1.65 & $\ldots$ \\
\hline$T_{\mathrm{d}}[\mathrm{K}]$ & $\ldots$ & 18.5 & $\ldots$ \\
\hline \multirow[t]{2}{*}{$\tau_{250}$} & $\ldots$ & $9.4 \times 10^{-4}$ & $\ldots$ \\
\hline & & $\rho$ Ophiuchi & \\
\hline$N_{\mathrm{H}}\left[10^{21} \mathrm{~cm}^{-2}\right]$ & 18.2 & 0.4 & 0.4 \\
\hline$n_{\mathrm{H}}\left[\mathrm{cm}^{-3}\right]$ & $2 \times 10^{4}$ & 200 & 0.5 \\
\hline$z[\mathrm{pc}]$ & 0.3 & 0.6 & $\ldots$ \\
\hline$G_{0}$ & 0.4 & 400 & $\ldots$ \\
\hline$T[\mathrm{~K}]$ & 20 & $10^{3}$ & $8 \times 10^{3}$ \\
\hline$x_{\mathrm{H}}[\mathrm{ppm}]$ & 9.2 & 373 & $10^{6}$ \\
\hline$x_{\mathrm{C}}[\mathrm{ppm}]$ & $<1$ & 100 & $\ldots$ \\
\hline$y$ & 1 & 0.1 & $\ldots$ \\
\hline$a_{0}[\mathrm{~nm}]$ & 0.60 & 0.38 & $\ldots$ \\
\hline$b_{\mathrm{C}}[\mathrm{ppm}]$ & 65 & 50 & $\ldots$ \\
\hline$\beta$ & $\ldots$ & 1.75 & $\ldots$ \\
\hline$T_{\mathrm{d}}[\mathrm{K}]$ & $\ldots$ & 20.7 & $\ldots$ \\
\hline$\tau_{250}$ & $\ldots$ & $3.2 \times 10^{-3}$ & $\ldots$ \\
\hline
\end{tabular}

Notes. $x_{\mathrm{H}}$ and $x_{\mathrm{C}}$ are the abundances of $\mathrm{H}^{+}$and $\mathrm{C}^{+} ; y$ is the molecular fraction $2 n_{\mathrm{H}_{2}} / n_{\mathrm{H}} ; a_{0}$ is the centroid of the PAH size distribution; $b_{\mathrm{C}}$ is the PAH abundance of carbon in PAHs; and $z$ is the depth of the emission region along the line of sight. The radiation field is that of Mathis et al. (1983) multiplied by the factor $G_{0}$. The ionised gas accounts for the free-free emission but does not contribute to the spinning dust emission.

the size of $\mathrm{PAHs}^{3}$. The largest PAHs have the lowest emissivity. In low temperature gas $\left(G_{0}<3\right.$ and $\left.n_{\mathrm{H}}>30 \mathrm{~cm}^{-3}\right)$, the gasgrain collisions dominate the excitation and the damping of the grain rotation. Thus the spinning dust spectrum becomes sensitive to the abundance of the major ions, $\mathrm{H}^{+}$and $\mathrm{C}^{+}$, denoted $x_{\mathrm{H}}$ and $x_{\mathrm{C}}$ respectively. To compute the spinning dust emissivity, we use SPDUST ${ }^{4}$ in the case 2 of Silsbee et al. (2011). Rather than fitting all the spinning dust parameters on the observed spectrum, we derive realistic values from observations, and fit the amplitude to the spectrum. We derive $x_{\mathrm{H}}$ from the ionisation balance of hydrogen assuming a standard cosmic-ray ionisation rate of $5 \times 10^{-17} \mathrm{~s}^{-1} \mathrm{H}^{-1}$ (Williams et al. 1998; Wolfire et al. 2003). Conversely, $x_{\mathrm{C}}$ cannot be estimated so readily because $\mathrm{C}$ undergoes reactions with $\mathrm{H}_{2}$ and PAHs (Röllig et al. 2006; Wolfire et al. 2008); we therefore take $x_{\mathrm{C}}$ as a free parameter. The size distribution of PAHs is assumed to be a log-normal of centroid $a_{0}$ and width $\sigma=0.4$. The abundance of PAHs is set by $b_{\mathrm{C}}$, the abundance of carbon locked up in PAHs.

\footnotetext{
3 We assume here the electric dipole moment to be as in Draine \& Lazarian (1998), a prescription also shown to be compatible with the AME extracted from WMAP data (Ysard et al. 2010).

${ }^{4}$ http://www.tapir.caltech.edu/ yacine/spdust/spdust. html
} 
Now we describe the physical conditions within our two regions, the Perseus and $\rho$ Ophiuchi molecular clouds, as derived from observations, and how we take them into account in our modelling (see Table 4). For both regions, the freefree contribution is that of the $\mathrm{H}_{\text {II }}$ region and the thermal dust emission is described as a modified blackbody, $I_{v}=$ $\tau_{250}(\lambda / 250 \mu \mathrm{m})^{-\beta} B_{v}(T)$ where $\tau_{250}$ is the dust opacity at $250 \mu \mathrm{m}, T$ the dust temperature (see Table 4), and $B_{v}$ is the blackbody brightness. We do not include the contribution of the ionised gas to the spinning dust emission because of its low density and also because PAHs have been shown to be destroyed efficiently in such environments (Lebouteiller et al. 2007, 2011). In addition, we note that the current SPDUST code does not include ionising photons $(h v \geq 13.6 \mathrm{eV})$, which are important for the PAH charge and IR emission.

\subsection{Perseus molecular cloud}

At the degree scale, the Perseus line of sight intersects a molecular cloud surrounding the $\mathrm{H}$ in region associated with the HD 278942 B0 V star. Ridge et al. (2006) argued that this ionised shell has a density of $\sim 1 \mathrm{~cm}^{-3}$. The gas density and temperature of the molecular component are taken from the analysis of observed $C_{2}$ lines carried out by Iglesias-Groth $(2011)^{5}$. The parameters of our model, which provides a good match to the AME, are given in Table 4, and the corresponding emission is shown in Fig. 5. We note that the depth, $z$, of the emissive region along the sightline is compatible with determinations at smaller scales (Ridge et al. 2006). The spinning dust emission is dominated by the contribution of the dense gas; the low-density atomic gas appears to play only a minor role. This is consistent with the results of Planck Collaboration (2011q), which show that, on large scales, there is bright AME associated with molecular clouds traced by $\mathrm{CO}$ everywhere in the Galaxy, while the association with the diffuse and atomic phase correlated with $\mathrm{H}_{\mathrm{I}}$ is less clear.

In contrast to previous work where $x_{\mathrm{H}}=0$ was assumed in molecular gas, our ionisation balance of $\mathrm{H}$ gives $x_{\mathrm{H}} \sim 3 \times 10^{-5}$, a change particularly important for Perseus. We take $x_{\mathrm{C}}$ to be 1 ppm: at this level the $\mathrm{C}^{+}$ion has no influence on the spinning dust emission. Adopting larger sizes for the PAHs $\left(a_{0}=\right.$ $0.58 \mathrm{~nm}$ ) than those used in previous spinning dust models (but comparable to the diffuse interstellar medium $(0.64 \mathrm{~nm})$; Compiègne et al. 2011), the molecular gas accounts well for the AME.

\subsection{The $\rho$ Ophiuchi molecular cloud}

The $\rho$ Ophiuchi spectrum includes contributions from a photodissociated interface region (PDR) and the ionised gas associated with the star HD 147889. We note that IR observations have shown that the PAH emission arises from dense irradiated interfaces (Abergel et al. 2002; Habart et al. 2005). As constrained by Habart et al. (2003), the PDR has a density gradient between the star and the molecular cloud. For the present preliminary model, we represent this interface with two effective media at low $\left(200 \mathrm{~cm}^{-3}\right)$ and high $\left(2 \times 10^{4} \mathrm{~cm}^{-3}\right)$ densities irradiated by strong and weak radiation fields. In the more excited $\rho$ Ophiuchi region we are able to match the AME by requiring: (a) an attenuated radiation field in the dense gas; and (b) smaller and

\footnotetext{
5 A single high resolution measurement may not be representative of the line-of-sight on degree scales, since the density and temperature may vary considerably across the beam.
}

less abundant PAHs in stronger radiation fields, in line with recent work suggesting that PAHs are photoevaporated from larger carbonaceous grains (Berné et al. 2007; Compiègne et al. 2008; Velusamy \& Langer 2008). The PAH abundances we assume for both regions are within the range found by Habart et al. (2003). The fit is shown in Fig. 9 and the parameters of our model are summarised in Table 4.

We note, however, that the determination of $a_{0}, G_{0}$ and $n_{\mathrm{H}}$ is degenerate in the present modelling; you can create almost identical spinning dust curves by varying these parameters in different ways (see, e.g., Ali-Haïmoud et al. 2009). Lifting this degeneracy involves treating the radiative transfer along with the spinning dust motion. The present results are therefore suggestive only and we emphasise that the physical conditions of our models are derived from scales much smaller than the resolution of our observations. The previous discussion also indicates that quantitative model spectra can only be obtained from a consistent treatment of the gas state, the radiative transfer, and the spinning motion of dust grains. Finally, a combination of multifrequency data, including detailed IR measurements, is clearly needed to extract detailed information about the environment and the dust grains.

\section{New regions of anomalous emission}

We have searched the Planck maps for new regions of AME on the scale of $\sim 3^{\circ}$ or smaller. To do this, one must suppress the other emission components that contribute to the frequencies where AME is the strongest ( $20-60 \mathrm{GHz})$. Numerous methods can be used (e.g., Leach et al. 2008; Delabrouille \& Cardoso 2009). However, we have taken a simplistic approach to identify bright AME regions. We subtracted a simple spatial model of known emission mechanisms (synchrotron, free-free, and thermal dust) using extrapolations of existing templates from observations or theoretical expectations, and then examined the locations of the remaining emission in more detail. We used maps smoothed to $1^{\circ}$, with HEALPix $N_{\text {side }}=256$ (Górski et al. 2005).

We subtracted the synchrotron emission from the CMBsubtracted Planck maps using the $408 \mathrm{MHz}$ map (Haslam et al. 1982), while the $1420 \mathrm{MHz}$ (Reich 1982; Reich \& Reich 1986; Reich et al. 2001) and the $2326 \mathrm{MHz}$ (Jonas et al. 1998) maps were used in conjunction with $408 \mathrm{MHz}$ data to determine the spectral index. Brightness temperature spectral indices were restricted to lie in the range $-2.0>\beta>-4.0\left(T \propto v^{\beta}\right)$. For the gap region where no $1420 / 2326 \mathrm{MHz}$ data exist, we assume $\beta=-3.0$. We used versions of the maps that have had no point sources subtracted (Platania et al. 2003) so as to allow direct comparison with the Planck data. For free-free emission, we use the $\mathrm{H} \alpha$ map of Dickinson et al. (2003), which is based on WHAM (Haffner et al. 2003) and SHASSA (Gaustad et al. 2001) data corrected for dust absorption. The map is also used to correct the synchrotron maps for free-free emission, assuming an average electron temperature of $8000 \mathrm{~K}$. The Rayleigh-Jeans tail of the thermal (vibrational) dust component is determined from the Planck maps at 143 and $545 \mathrm{GHz}$, thereby avoiding significant CO contamination. The $545 \mathrm{GHz}$ data were used for normalisation and the $143 \mathrm{GHz}$ data were used for the spectral index of the power-law. This was constrained to lie between 0 and +3 to avoid extrapolation artefacts in some areas at high latitudes where the uncertainty on the spectral index is large.

The full-sky map of the residuals from the $28.5 \mathrm{GHz}$ Planck LFI data is shown in Fig. 10. Artefacts from the subtraction are present. The division between the 1420 and the 


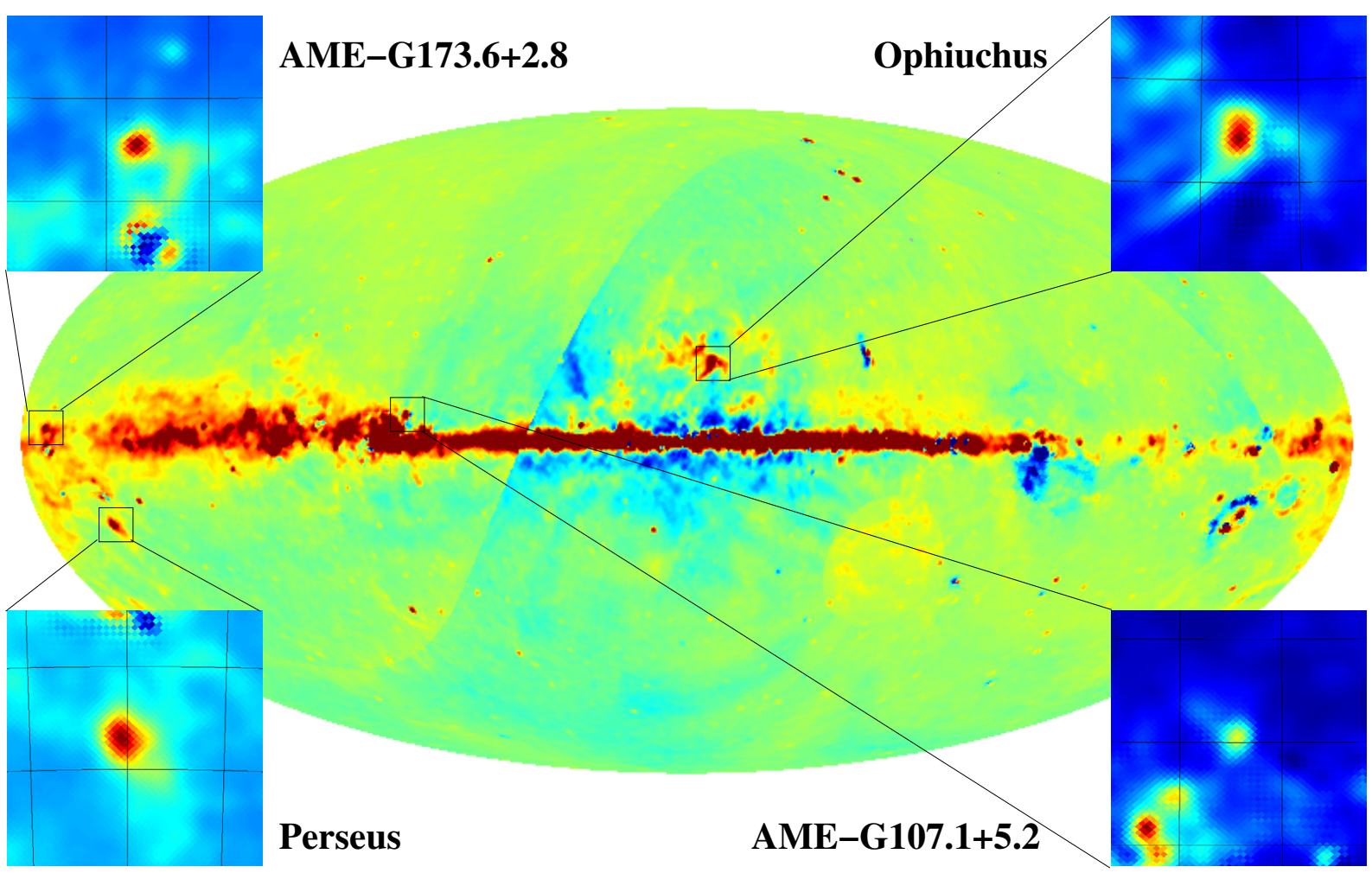

Fig. 10. Residuals in the full sky Planck LFI $28.5 \mathrm{GHz} 1^{\circ}$ smoothed map after subtraction of synchrotron, free-free and thermal dust emission (see text). $12.5 \times 12.5$ Cut out maps are shown for the Perseus and $\rho$ Ophiuchi molecular clouds, and the two new regions of AME, AME-G107.1+5.2 and AME-G173.6+2.8. The graticule spacing is $5^{\circ}$ in Galactic coordinates.

$2326 \mathrm{MHz}$ maps is evident as well as the lack of observations around the south celestial pole. Other large-scale features are also present. These are extended features and hence do not affect our search for compact areas of AME. A large number of AME candidates are evident in the map, including the strong Perseus and $\rho$ Ophiuchi AME regions described in Sects. 4 and 5. We examined $\sim 50$ regions in detail.

The next step in identifying new AME regions is to construct a spectrum for each candidate, using aperture photometry. In each case there is strong thermal dust and free-free emission indicative of an associated $\mathrm{H}$ II region. We now illustrate the results of our search in two of the compact regions which show excess emission in the $\sim 20-60 \mathrm{GHz}$ range.

\subsection{AME-G173.6+2.8}

AME-G173.6+2.8 $\left(05^{\mathrm{h}} 41^{\mathrm{m}},+35^{\circ} 51^{\prime} \mathrm{J} 2000.0\right)$ is a strong, compact region of dust emission towards the Galactic anticentre. The H II region S235 (Sharpless 1959) lies within this area. It is a region of massive star formation with a young stellar object at its centre, and it has several compact radio components within several arcmin (Nordh et al. 1984; Felli et al. 2006). Radio recombination line observations indicate a radial velocity of $-24.5 \pm 0.7 \mathrm{~km} \mathrm{~s}^{-1}$ (Paladini et al. 2003). S235 lies at a distance of $1.8 \mathrm{kpc}$ and is therefore within the Perseus spiral arm.

Maps of the region at a range of frequencies are shown at the observing resolution of each in Fig. 11. The AME is strong at $28.5 \mathrm{GHz}$ while the dust distribution at the higher Planck frequencies shows the small-scale structure of the dust within the $28.5 \mathrm{GHz}$ beam. It can be seen that the $\mathrm{H} \alpha$ at the $6^{\prime}$ resolution of Finkbeiner (2003) is offset from the dust, most likely due to absorption by the dust.
The spectrum of the 1.3 degree (COSMOSOMAS) region centred on AME-G173.6+2.8 is shown in Fig. 12. The radio flux densities are at $820 \mathrm{MHz}$ (Berkhuijsen 1972), $1.4 \mathrm{GHz}$ (Reich 1982) and 11-17 GHz (COSMOSOMAS). The spectrum and residuals of the region are derived using the COSMOSOMAS filtering method described in Sect. 4.2.

The spectrum is well-fitted using a combination of thermal dust (with $T=19.6 \pm 0.6 \mathrm{~K}$ and an emissivity index of $\beta=1.54 \pm$ $0.07)$, free-free emission $\left(E M=(2.47 \pm 0.13) \times 10^{3} \mathrm{~cm}^{-6} \mathrm{pc}\right)$ and spinning dust (Draine \& Lazarian $1998 \mathrm{CNM}$ model with $\left.N_{\mathrm{H}}=(2.7 \pm 0.3) \times 10^{22} \mathrm{~cm}^{-2}\right)$, with a small CMB contribution of $\Delta T_{\mathrm{CMB}}=130 \pm 40 \mu \mathrm{K}$. The residual spectrum shows the signature of spinning dust peaking in the frequency range $20-30 \mathrm{GHz}$, detected with a significance level of $6.4 \sigma$. The main uncertainty in the AME spectrum is the level of free-free emission to be subtracted. As shown, the spinning dust emission in the $20-30 \mathrm{GHz}$ region is $\sim 2$ times the free-free emission; the uncertainty is $<10$ $20 \%$. The current spectral coverage defines the peak of the spectrum and provides useful constraints in modelling the AME. An ultracompact $\mathrm{H}$ II region, producing free-free emission that is optically thick at frequencies $<10 \mathrm{GHz}$, could possibly contribute to the flux density at $>10 \mathrm{GHz}$. However, it cannot easily account for the shape at high $(\gtrsim 40 \mathrm{GHz})$ frequencies.

\section{2. $A M E-G 107.1+5.2$}

AME-G107.1+5.2 $\left(22^{\mathrm{h}} 22^{\mathrm{m}},+63^{\circ} 23^{\prime} \mathrm{J} 2000.0\right)$ is a dust complex containing the star-forming H II region S140 (Sharpless 1959) with $V_{\mathrm{LSR}}=-8.5 \pm 1.0 \mathrm{~km} \mathrm{~s}^{-1}$. The region lies at the edge of the Cepheus bubble (Patel et al. 1998) at a distance of 800 pc.

Maps of the region are shown in Fig. 13 using the same format as in Fig. 11. Dust is widespread over the region but with 

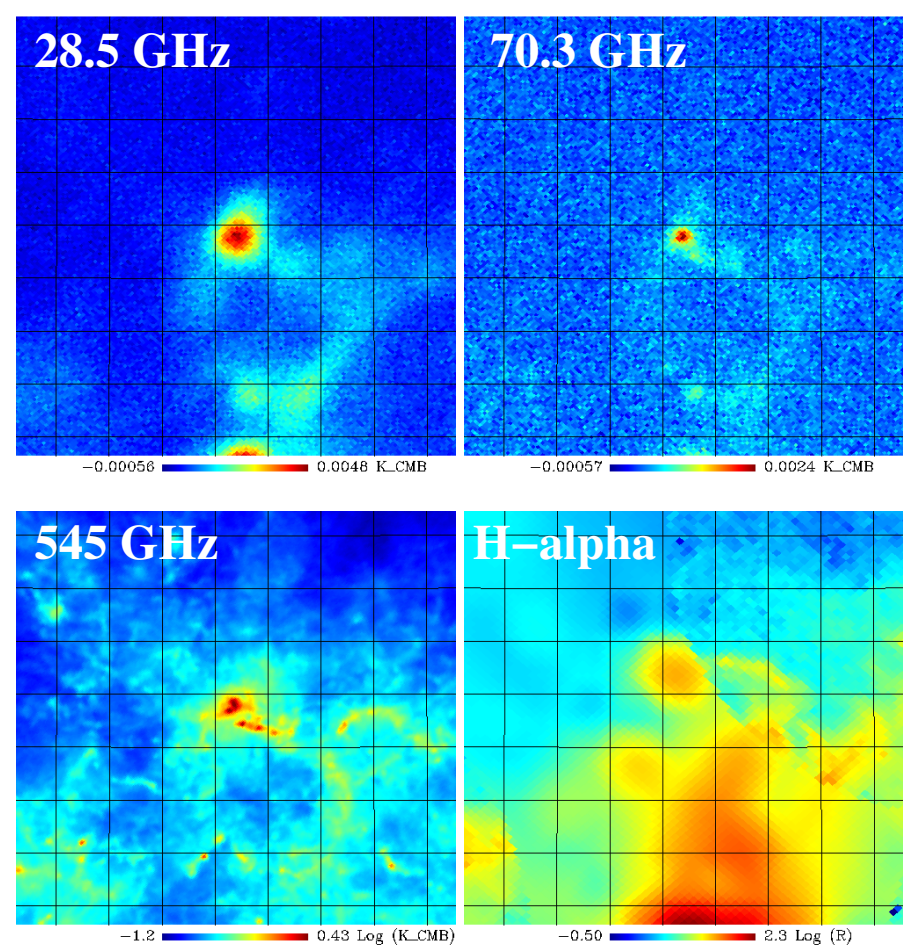

Fig. 11. Maps of the AME-G173.6+2.8 region: $28.5 \mathrm{GHz}$ (linear scale); $70.3 \mathrm{GHz}$ (linear scale); $545 \mathrm{GHz}$ (logarithmic scale); and $\mathrm{H} \alpha$ (logarithmic scale). The maps are $8^{\circ} \times 8^{\circ}$ and the graticule spacing is $1^{\circ}$ in Galactic coordinates.

a concentration at the position of the AME. There are multiple dust components within the $28.5 \mathrm{GHz}$ beam. There is a small offset of the $28.5 \mathrm{GHz}$ feature of $\approx 15^{\prime}$ to lower latitudes and higher longitudes than the brightest feature in the dust maps. This indicates a higher radio emissivity of the dust in this offset position. There is also an offset in the $\mathrm{H} \alpha$ emission, again possibly due to absorption by the associated dust.

The spectrum estimated for AME-G107.1+5.2 and the residual spectrum are shown in Fig. 14. The least-squares fit to the free-free emission $\left(E M=150 \pm 39 \mathrm{~cm}^{-6} \mathrm{pc}\right)$, thermal dust $\left(T_{\mathrm{d}}=19.0 \pm 2.6 \mathrm{~K}, \beta=2.04 \pm 0.45\right)$, spinning dust (Draine \& Lazarian 1998 WIM model with $\left.N_{\mathrm{H}}=(1.76 \pm 0.44) \times 10^{21} \mathrm{~cm}^{-2}\right)$, and $\mathrm{CMB}\left(\Delta T_{\mathrm{CMB}}=75 \pm 38 \mu \mathrm{K}\right)$ is well-defined. The AME is detected at a significance of $4.8 \sigma$ with a shape that is consistent with spinning dust. However, an ultracompact $\mathrm{H}$ II region producing optically thick free-free at frequencies below $10 \mathrm{GHz}$ may also be contributing.

\subsection{Assessment of the search for new AME regions}

We have shown that it is possible to detect bright compact AME regions in the Planck data using a rather simplistic selection process. In order to verify the identifications it is necessary to add ancillary data at radio and FIR frequencies. Now that identifications have been made, new ancillary data covering a larger frequency range will be sought. Further development of the search algorithms will also be worthwhile.

The strength of AME relative to the free-free and thermal dust emission in these two regions can be compared with the values for $\mathrm{H}$ II regions obtained by Todorović et al. (2010) in the VSA survey of the $l=27^{\circ}-47^{\circ}$ section of the Galactic plane. The latter survey found the ratio of $33 \mathrm{GHz} \mathrm{AME}$ to
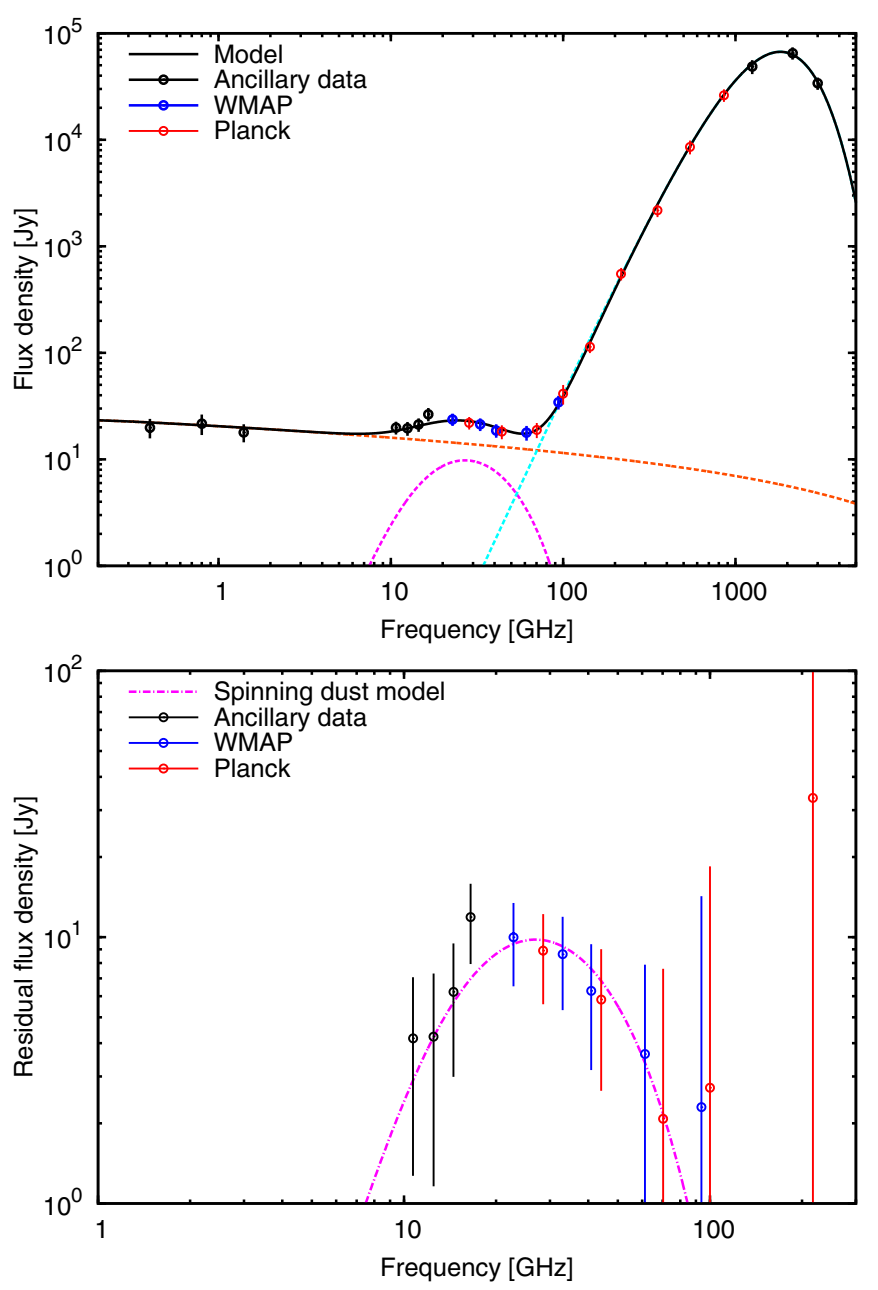

Fig. 12. The spectrum (top) and residuals (bottom) for AMEG173.6+2.8 after removing free-free emission (orange dashed line), thermal dust emission (light blue dashed line) and CMB anisotropies (not visible). A spinning dust model is shown as a magenta dot-dashed line.

free-free flux density to be $0.40 \pm 0.11$; the corresponding values for AME-G173.6+2.8 and AME-G107.1+5.2 are 0.67 and 1.4, respectively. In the case of the ratio of $33 \mathrm{GHz}$ AME to $100 \mu \mathrm{m}$ thermal dust emission, the VSA survey value was $1.1 \times 10^{-4}$ (or $2 \times 10^{-4}$ at the $15 \mathrm{GHz}$ peak of the spectrum), while the AME-G173.6+2.8 and the AME-G107.1+5.2 values are somewhat larger at $3.3 \times 10^{-4}$ and $3.8 \times 10^{-4}$, respectively. It is however not surprising that the $30 \mathrm{GHz}$ AME is stronger in the two Planck H II regions than in the VSA H II regions, because the former were selected as the brightest AME sources in the Planck maps. The contribution from ultracompact $\mathrm{H}_{\text {II }}$ regions (Wood \& Churchwell 1989) may also be a significant contributor to the AME at low Galactic latitudes.

Finally, we note that the derived dust temperature for these $\mathrm{H}_{\mathrm{II}}$ regions, at $T_{\mathrm{d}} \approx 19 \mathrm{~K}$, is closer to that of the diffuse interstellar medium than to the warm dust $\left(T_{\mathrm{d}} \sim 30-50 \mathrm{~K}\right)$ usually found surrounding OB stars (e.g., Wood \& Churchwell 1989). The dust observed along the line of sight at $\sim 1^{\circ}$ resolution is likely to be a mixture of populations, including higher density regions (e.g., molecular clouds) that could dominate the signal on these scales. This may explain the lower average temperature and the presence of significant AME in these sightlines. 

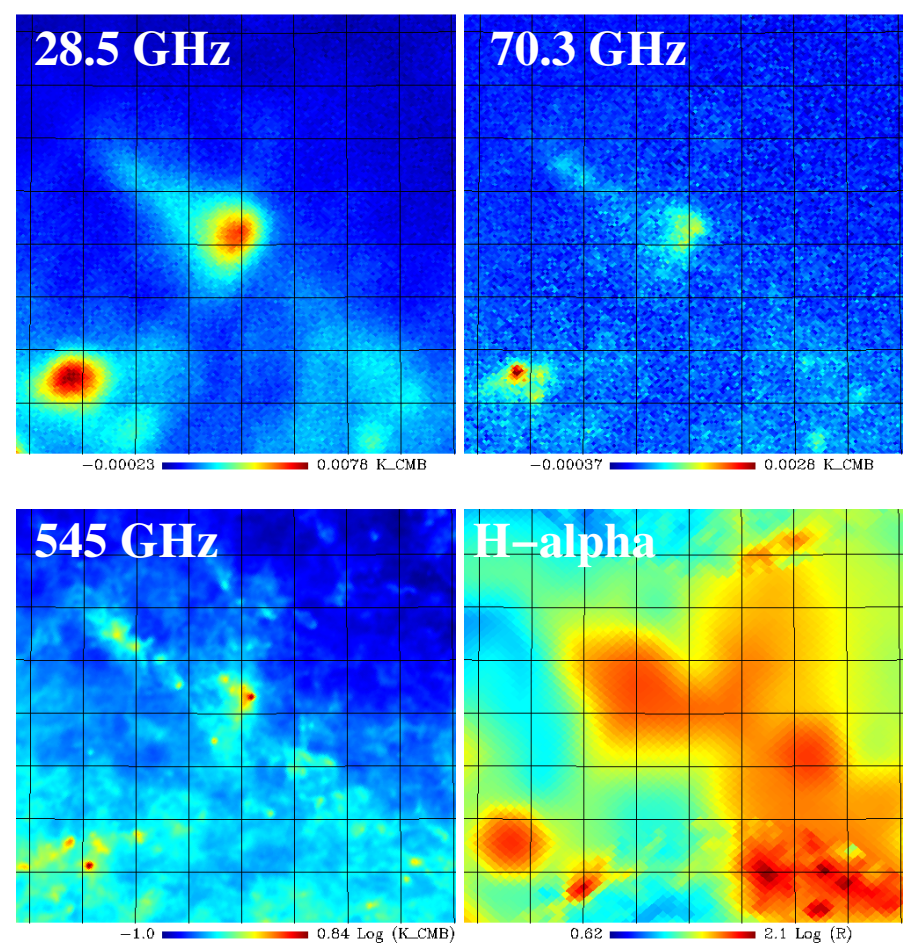

Fig. 13. Maps of the AME-G107.1+5.2 region: $28.5 \mathrm{GHz}$ (linear scale); $70.3 \mathrm{GHz}$ (linear scale); $545 \mathrm{GHz}$ (logarithmic scale); and $\mathrm{H} \alpha$ (logarithmic scale). The maps are $8^{\circ} \times 8^{\circ}$ and the graticule spacing is $1^{\circ}$ in Galactic coordinates.

As selection procedures develop, we expect many AME regions to be discovered in a range of physical environments, which will provide data necessary to acquire a full understanding of the AME emission mechanism.

\section{Conclusions}

Filling in the gap between earlier radio and FIR measurements, the frequency coverage of Planck has provided a unique opportunity to establish comprehensive spectra of regions of AME. Planck has revealed the high frequency side of the spectral peak. The new spectra are the basis for understanding the emission mechanism and the environment in which it occurs. The evidence from the present observations strongly favours the spinning dust mechanism (electric dipole radiation). Planck provides a rich data set that can be used as a basis for developing a realistic understanding of the AME mechanism in a range of Galactic environments.

The two best-studied AME sources that have extensive ancillary data are the Perseus and $\rho$ Ophiuchi molecular clouds. In each, the spectrum is well fitted by free-free, thermal dust, and spinning dust, with a small contribution from the CMB. Spinning dust provides a good fit to the microwave $(10-100 \mathrm{GHz})$ part of the spectrum, which peaks at $\approx 30 \mathrm{GHz}$.

Theoretical spinning dust spectra are presented for a physical model consisting of molecular and atomic states. It is possible to derive physical parameters that are consistent with the environment and still provide a good fit to the data. Using parameters constrained at smaller angular scales, the $20-40 \mathrm{GHz}$ AME peak in Perseus is well explained with spinning dust emission arising from dense, molecular gas $\left(n_{\mathrm{H}}>200 \mathrm{~cm}^{-3}\right)$ subjected to a few times the typical interstellar radiation field.
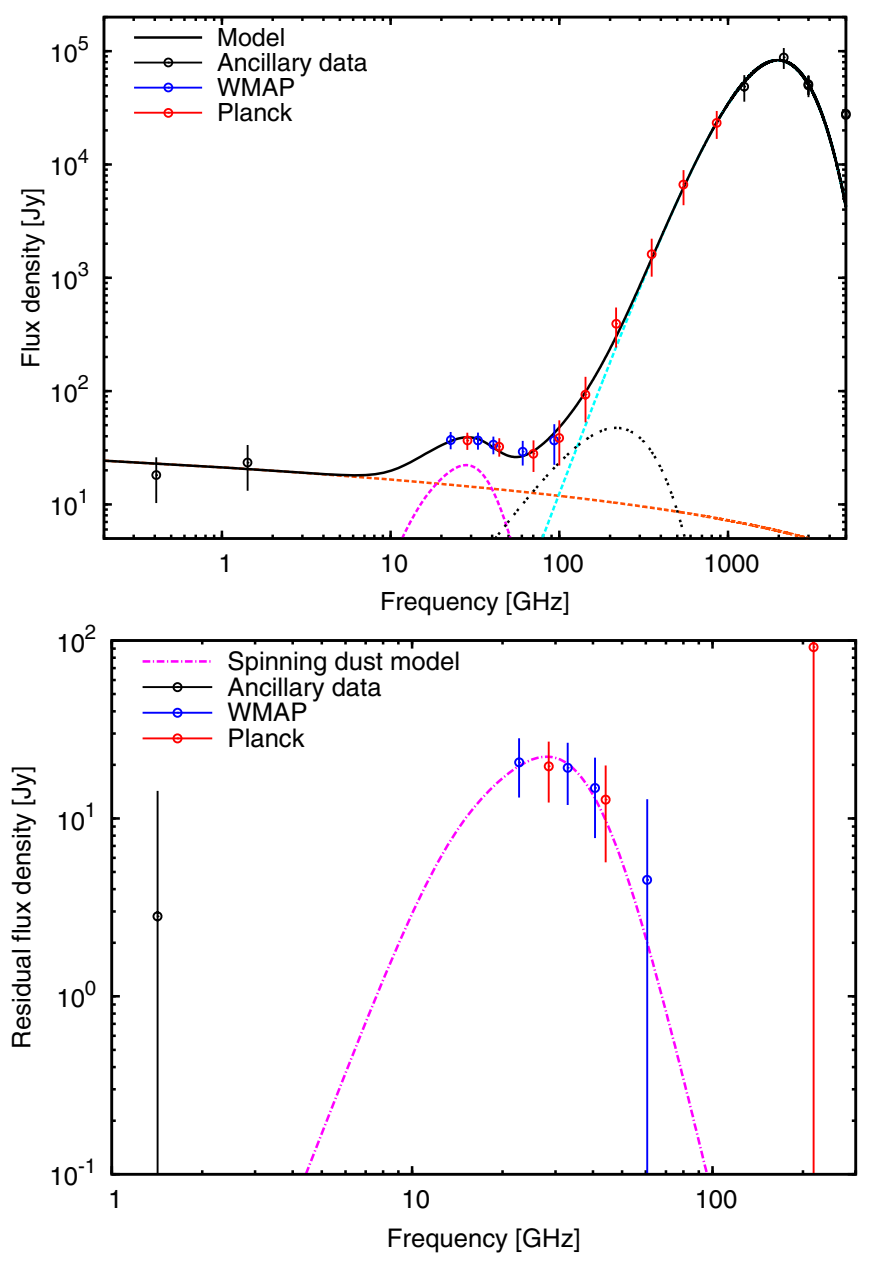

Fig. 14. The spectrum (top) and residuals (bottom) for AMEG107.1+5.2 after removing free-free (orange dashed line), thermal dust emission (light blue dashed line) and CMB anisotropies (black doubledotted line). A spinning dust model is shown as a magenta dot-dashed line.

Low-density gas is only a minor contributor to AME in Perseus; however, in $\rho$ Ophiuchi, although dense gas accounts for the peak at $\approx 30 \mathrm{GHz}$, irradiated low-density atomic gas may be contributing in the range $50-100 \mathrm{GHz}$. The picture seems to be that smaller PAHs are found in PDRs $\left(G_{0}>100\right)$, as suggested by recent Spitzer observations. However, the determination of the PAH size is degenerate with that of $n_{\mathrm{H}}$ and $G_{0}$ and quantitative conclusions will only be obtained from consistent modelling of the gas state, radiative transfer, and spinning dust. At this level of modelling it is not possible to constrain the electric dipole moment of PAHs. Future work to carry out more detailed modelling is required.

Our preliminary search for new AME regions in the Planck data has revealed many candidates. They were uncovered by subtracting synchrotron, free-free and thermal dust emission based on the usual spatial templates. Two of the new candidate regions that show AME at $>5 \sigma$ significance have spectra consistent with a spinning dust spectral shape. Additional multifrequency data (e.g., such as the $5 \mathrm{GHz}$ C-Band All-Sky Survey, $\mathrm{C}^{-B_{A S S}}{ }^{6}$; King et al. 2010) as well as high-resolution observations will be needed to understand the detailed structure of the AME in these regions.

${ }^{6}$ http://www.astro.caltech.edu/cbass/ 
Acknowledgements. We thank the referee, Doug Finkbeiner, for useful comments. We thank Justin Jonas for providing the $2326 \mathrm{MHz}$ HartRAO map. We acknowledge the use of the MPIfR Survey Sampler website at http://www. mpifr-bonn.mpg.de/survey.html. We acknowledge the use of the Legacy Archive for Microwave Background Data Analysis (LAMBDA); support for LAMBDA is provided by the NASA Office of Space Science. This research has made use of the NASA/IPAC Extragalactic Database (NED) which is operated by the Jet Propulsion Laboratory, California Institute of Technology, under contract with the National Aeronautics and Space Administration. This research makes use of the SIMBAD database, operated at CDS, Strasbourg, France.

The Planck Collaboration acknowledges the support of: ESA; CNES and CNRS/INSU-IN2P3-INP (France); ASI, CNR, and INAF (Italy); NASA and DoE (USA); STFC and UKSA (UK); CSIC, MICINN and JA (Spain); Tekes, AoF and CSC (Finland); DLR and MPG (Germany); CSA (Canada); DTU Space (Denmark); SER/SSO (Switzerland); RCN (Norway); SFI (Ireland); FCT/MCTES (Portugal); and DEISA (EU). A detailed description of the Planck Collaboration and a list of its members can be found at http://www.rssd. esa .int/index .php?project=PLANCK\&page=Planck_Collaboration.

\section{References}

Abergel, A., Bernard, J. P., Boulanger, F., et al. 2002, A\&A, 389, 239 Ali-Haïmoud, Y., Hirata, C. M., \& Dickinson, C. 2009, MNRAS, 395, 1055 Banday, A. J., Dickinson, C., Davies, R. D., Davis, R. J., \& Górski, K. M. 2003, MNRAS, 345, 897

Bennett, C. L., Hill, R. S., Hinshaw, G., et al. 2003, ApJS, 148, 97

Berkhuijsen, E. M. 1972, A\&AS, 5, 263

Berné, O., Joblin, C., Deville, Y., et al. 2007, A\&A, 469, 575

Bersanelli, M., Mandolesi, N., Butler, R. C., et al. 2010, A\&A, 520, A4

Casassus, S., Cabrera, G. F., Förster, F., et al. 2006, ApJ, 639, 951

Casassus, S., Dickinson, C., Cleary, K., et al. 2008, MNRAS, 391, 1075

Castellanos, P., Casassus, S., Dickinson, C., et al. 2011, MNRAS, 411, 1137

Cernicharo, J., Bachiller, R., \& Duvert, G. 1985, A\&A, 149, 273

Compiègne, M., Abergel, A., Verstraete, L., \& Habart, E. 2008, A\&A, 491, 797

Compiègne, M., Verstraete, L., Jones, A., et al. 2011, A\&A, 525, A103

Condon, J. J., Cotton, W. D., Greisen, E. W., et al. 1998, AJ, 115, 1693

Dame, T. M., Hartmann, D., \& Thaddeus, P. 2001, ApJ, 547, 792

Davies, R. D., Lasenby, A. N., Watson, R. A., et al. 1987, Nature, 326, 462

Davies, R. D., Dickinson, C., Banday, A. J., et al. 2006, MNRAS, 370, 1125 de Oliveira-Costa, A., Kogut, A., Devlin, M. J., et al. 1997, ApJ, 482, L17

de Oliveira-Costa, A., Tegmark, M., Gutierrez, C. M., et al. 1999, ApJ, 527, L9

de Oliveira-Costa, A., Tegmark, M., Davies, R. D., et al. 2004, ApJ, 606, L89

de Zeeuw, P. T., Hoogerwerf, R., de Bruijne, J. H. J., Brown, A. G. A., \& Blaauw, A. $1999, \mathrm{AJ}, 117,354$

Delabrouille, J., \& Cardoso, J. 2009, in Data Analysis in Cosmology, ed.

V. J. Martínez, E. Saar, E. Martínez-González, \& M.-J. Pons-Bordería, Lect.

Notes Phys. (Berlin: Springer Verlag), 665, 159

Dickinson, C., Davies, R. D., \& Davis, R. J. 2003, MNRAS, 341, 369

Dickinson, C., Davies, R. D., Allison, J. R., et al. 2009, ApJ, 690, 1585

Dickinson, C., Casassus, S., Davies, R. D., et al. 2010, MNRAS, 407, 2223

Dobler, G., \& Finkbeiner, D. P. 2008, ApJ, 680, 1222

Dobler, G., Draine, B., \& Finkbeiner, D. P. 2009, ApJ, 699, 1374

Draine, B. T., \& Lazarian, A. 1998, ApJ, 508, 157

Draine, B. T., \& Lazarian, A. 1999, ApJ, 512, 740

Encrenaz, P. J. 1974, ApJ, 189, L135

Erickson, W. C. 1957, ApJ, 126, 480

Evans, II, N. J., Allen, L. E., Blake, G. A., et al. 2003, PASP, 115, 965

Felli, M., Massi, F., Robberto, M., \& Cesaroni, R. 2006, A\&A, 453, 911

Fernández-Cerezo, S., Gutiérrez, C. M., Rebolo, R., et al. 2006, MNRAS, 370, 15

Finkbeiner, D. P. 2003, ApJS, 146, 407

Finkbeiner, D. P. 2004, ApJ, 614, 186

Finkbeiner, D. P., Davis, M., \& Schlegel, D. J. 1999, ApJ, 524, 867

Finkbeiner, D. P., Schlegel, D. J., Frank, C., \& Heiles, C. 2002, ApJ, 566, 898

Finkbeiner, D. P., Langston, G. I., \& Minter, A. H. 2004, ApJ, 617, 350

Gallegos, J. E., Macías-Pérez, J. F., Gutiérrez, C. M., et al. 2001, MNRAS, 327, 1178

Gaustad, J. E., McCullough, P. R., Rosing, W., \& Van Buren, D. 2001, PASP, 113,1326

Gold, B., Odegard, N., Weiland, J. L., et al. 2011, ApJS, 192, 15

Górski, K. M., Hivon, E., Banday, A. J., et al. 2005, ApJ, 622, 759

Habart, E., Boulanger, F., Verstraete, L., et al. 2003, A\&A, 397, 623

Habart, E., Abergel, A., Walmsley, C. M., Teyssier, D., \& Pety, J. 2005, A\&A,

437, 177

Haffner, L. M., Reynolds, R. J., Tufte, S. L., et al. 2003, ApJS, 149, 405

Haslam, C. G. T., Salter, C. J., Stoffel, H., \& Wilson, W. E. 1982, A\&AS, 47, 1

Hauser, M. G., Arendt, R. G., Kelsall, T., et al. 1998, ApJ, 508, 25
Hildebrandt, S. R., Rebolo, R., Rubiño-Martín, J. A., et al. 2007, MNRAS, 382, 594

Hoang, T., Draine, B. T., \& Lazarian, A. 2010, ApJ, 715, 1462

Iglesias-Groth, S. 2011, MNRAS, 411, 1857

Jarosik, N., Barnes, C., Bennett, C. L., et al. 2003, ApJS, 148, 29

Jarosik, N., Bennett, C. L., Dunkley, J., et al. 2011, ApJS, 192, 14

Jonas, J. L., Baart, E. E., \& Nicolson, G. D. 1998, MNRAS, 297, 977

Kaźmierczak, M., Schmidt, M. R., Bondar, A., \& Krełowski, J. 2010, MNRAS, 402, 2548

King, O. G., Copley, C., Davies, R., et al. 2010, in SPIE Conf. Ser., 7741

Kogut, A., Banday, A. J., Bennett, C. L., et al. 1996, ApJ, 464, L5

Kulesa, C. A., Hungerford, A. L., Walker, C. K., Zhang, X., \& Lane, A. P. 2005, ApJ, 625, 194

Lagache, G. 2003, A\&A, 405, 813

Lamarre, J., Puget, J., Ade, P. A. R., et al. 2010, A\&A, 520, A9

Leach, S. M., Cardoso, J., Baccigalupi, C., et al. 2008, A\&A, 491, 597

Leahy, J. P., Bersanelli, M., D’Arcangelo, O., et al. 2010, A\&A, 520, A8

Lebouteiller, V., Brandl, B., Bernard-Salas, J., Devost, D., \& Houck, J. R. 2007, ApJ, 665, 390

Lebouteiller, V., Bernard-Salas, J., Whelan, D. G., et al. 2011, ApJ, 728, 45

Leitch, E. M., Readhead, A. C. S., Pearson, T. J., \& Myers, S. T. 1997, ApJ, 486, L23

Liseau, R., White, G. J., Larsson, B., et al. 1999, A\&A, 344, 342

Lopez-Caraballo, C. H., Rubino-Martin, J. A., Rebolo, R., \& Genova-Santos, R. 2011, ApJ, 729, 25

Mandolesi, N., Bersanelli, M., Butler, R. C., et al. 2010, A\&A, 520, A3

Mathis, J. S., Mezger, P. G., \& Panagia, N. 1983, A\&A, 128, 212

Mennella, A., Butler, R. C., Curto, A., et al. 2011, A\&A, 536, A3

Miville-Deschênes, M., Ysard, N., Lavabre, A., et al. 2008, A\&A, 490, 1093

Murphy, E. J., Helou, G., Condon, J. J., et al. 2010, ApJ, 709, L108

Nordh, H. L., Fridlund, C. V. M., van Duinen, R. J., et al. 1984, A\&A, 131, 221

Paladini, R., Burigana, C., Davies, R. D., et al. 2003, A\&A, 397, 213

Patel, N. A., Goldsmith, P. F., Heyer, M. H., Snell, R. L., \& Pratap, P. 1998, ApJ, 507, 241

Perryman, M. A. C., Lindegren, L., Kovalevsky, J., et al. 1997, A\&A, 323, L49

Planck Collaboration 2011a, A\&A, 536, A1

Planck Collaboration 2011b, A\&A, 536, A2

Planck Collaboration 2011c, A\&A, 536, A7

Planck Collaboration 2011d, A\&A, 536, A8

Planck Collaboration 2011e, A\&A, 536, A9

Planck Collaboration 2011f, A\&A, 536, A10

Planck Collaboration 2011g, A\&A, 536, A11

Planck Collaboration 2011h, A\&A, 536, A12

Planck Collaboration 2011i, A\&A, 536, A13

Planck Collaboration 2011j, A\&A, 536, A14

Planck Collaboration 2011k, A\&A, 536, A15

Planck Collaboration 20111, A\&A, 536, A16

Planck Collaboration 2011m, A\&A, 536, A17

Planck Collaboration 2011n, A\&A, 536, A18

Planck Collaboration 2011o, A\&A, 536, A19

Planck Collaboration 2011p, A\&A, 536, A20

Planck Collaboration 2011q, A\&A, 536, A21

Planck Collaboration 2011r, A\&A, 536, A22

Planck Collaboration 2011s, A\&A, 536, A23

Planck Collaboration 2011t, A\&A, 536, A24

Planck Collaboration 2011u, A\&A, 536, A25

Planck Collaboration 2011v, The Explanatory Supplement to the Planck Early Release Compact Source Catalogue (ESA)

Planck Collaboration 2011w, A\&A, 536, A26

Planck HFI Core Team 2011a, A\&A, 536, A4

Planck HFI Core Team 2011b, A\&A, 536, A6

Platania, P., Burigana, C., Maino, D., et al. 2003, A\&A, 410, 847

Reich, W. 1982, A\&AS, 48, 219

Reich, P., \& Reich, W. 1986, A\&AS, 63, 205

Reich, P., \& Reich, W. 1988, A\&AS, 74, 7

Reich, W., \& Reich, P. 2009, in Cosmic Magnetic Fields: from Planets, to Stars and Galaxies, IAU Symp., 259, 603

Reich, P., Testori, J. C., \& Reich, W. 2001, A\&A, 376, 861

Ridge, N. A., Schnee, S. L., Goodman, A. A., \& COMPLETE Collaboration 2003, BAAS, 36, 580

Ridge, N. A., Schnee, S. L., Goodman, A. A., \& Foster, J. B. 2006, ApJ, 643, 932

Röllig, M., Ossenkopf, V., Jeyakumar, S., Stutzki, J., \& Sternberg, A. 2006, A\&A, 451, 917

Rosset, C., Tristram, M., Ponthieu, N., et al. 2010, A\&A, 520, A13

Scaife, A. M. M., Hurley-Walker, N., Green, D. A., et al. 2009, MNRAS, 400, 1394

Scaife, A. M. M., Nikolic, B., Green, D. A., et al. 2010, MNRAS, 406, L45 
Sharpless, S. 1959, ApJS, 4, 257

Silsbee, K., Ali-Haïmoud, Y., \& Hirata, C. M. 2011, MNRAS, 411, 2750

Tauber, J. A., Mandolesi, N., Puget, J., et al. 2010, A\&A, 520, A1

Tibbs, C. T., Watson, R. A., Dickinson, C., et al. 2010, MNRAS, 402, 1969

Todorović, M., Davies, R. D., Dickinson, C., et al. 2010, MNRAS, 406, 1629

van Dishoeck, E. F., \& Black, J. H. 1989, ApJ, 340, 273

Velusamy, T., \& Langer, W. D. 2008, AJ, 136, 602

Watson, R. A., Rebolo, R., Rubiño-Martín, J. A., et al. 2005, ApJ, 624, L89

Williams, J. P., Bergin, E. A., Caselli, P., Myers, P. C., \& Plume, R. 1998, ApJ, 503,689

Wolfire, M. G., McKee, C. F., Hollenbach, D., \& Tielens, A. G. G. M. 2003, ApJ, 587,278

Wolfire, M. G., Tielens, A. G. G. M., Hollenbach, D., \& Kaufman, M. J. 2008, ApJ, 680, 384

Wood, D. O. S., \& Churchwell, E. 1989, ApJS, 69, 831

Wootten, A., Evans, II, N. J., Snell, R., \& vanden Bout, P. 1978, ApJ, 225, L143

Wootten, A., Snell, R., \& Glassgold, A. E. 1979, ApJ, 234, 876

Young, K. E., Enoch, M. L., Evans, II, N. J., et al. 2006, ApJ, 644, 326

Ysard, N., \& Verstraete, L. 2010, A\&A, 509, A12

Ysard, N., Miville-Deschênes, M. A., \& Verstraete, L. 2010, A\&A, 509, L1

Zacchei, A., Maino, D., Baccigalupi, C., et al. 2011, A\&A, 536, A5

1 Aalto University Metsähovi Radio Observatory, Metsähovintie 114, 02540 Kylmälä, Finland

2 Agenzia Spaziale Italiana Science Data Center, c/o ESRIN, via Galileo Galilei, Frascati, Italy

3 Astroparticule et Cosmologie, CNRS (UMR7164), Université Denis Diderot Paris 7, Bâtiment Condorcet, 10 rue A. Domon et Léonie Duquet, Paris, France

4 Astrophysics Group, Cavendish Laboratory, University of Cambridge, J J Thomson Avenue, Cambridge CB3 OHE, UK

5 Atacama Large Millimeter/submillimeter Array, ALMA Santiago Central Offices, Alonso de Cordova 3107, Vitacura, Casilla 763 0355, Santiago, Chile

6 CITA, University of Toronto, 60 St. George St., Toronto, ON M5S 3H8, Canada

7 CNRS, IRAP, 9 Av. colonel Roche, BP 44346, 31028 Toulouse Cedex 4, France

8 California Institute of Technology, Pasadena, California, USA

9 Centre of Mathematics for Applications, University of Oslo, Blindern, Oslo, Norway

10 DAMTP, University of Cambridge, Centre for Mathematical Sciences, Wilberforce Road, Cambridge CB3 0WA, UK

11 DSM/Irfu/SPP, CEA-Saclay, 91191 Gif-sur-Yvette Cedex, France

12 DTU Space, National Space Institute, Juliane Mariesvej 30, Copenhagen, Denmark

13 Departamento de Física, Universidad de Oviedo, Avda. Calvo Sotelo s/n, Oviedo, Spain

14 Department of Astronomy and Astrophysics, University of Toronto, 50 Saint George Street, Toronto, Ontario, Canada

15 Department of Physics and Astronomy, University of British Columbia, 6224 Agricultural Road, Vancouver, British Columbia, Canada

16 Department of Physics, Gustaf Hällströmin katu 2a, University of Helsinki, Helsinki, Finland

17 Department of Physics, Princeton University, Princeton, New Jersey, USA

18 Department of Physics, Purdue University, 525 Northwestern Avenue, West Lafayette, Indiana, USA

19 Department of Physics, University of California, Berkeley, California, USA

20 Department of Physics, University of California, One Shields Avenue, Davis, California, USA

21 Department of Physics, University of California, Santa Barbara, California, USA

22 Department of Physics, University of Illinois at Urbana-Champaign, 1110 West Green Street, Urbana, Illinois, USA

23 Dipartimento di Fisica G. Galilei, Università degli Studi di Padova, via Marzolo 8, 35131 Padova, Italy

24 Dipartimento di Fisica, Università La Sapienza, P. le A. Moro 2, Roma, Italy
25 Dipartimento di Fisica, Università degli Studi di Milano, via Celoria, 16, Milano, Italy

26 Dipartimento di Fisica, Università degli Studi di Trieste, via A. Valerio 2, Trieste, Italy

27 Dipartimento di Fisica, Università di Ferrara, via Saragat 1, 44122 Ferrara, Italy

28 Dipartimento di Fisica, Università di Roma Tor Vergata, via della Ricerca Scientifica, 1, Roma, Italy

29 Discovery Center, Niels Bohr Institute, Blegdamsvej 17, Copenhagen, Denmark

30 Dpto. Astrofísica, Universidad de La Laguna (ULL), 38206 La Laguna, Tenerife, Spain

31 European Southern Observatory, ESO Vitacura, Alonso de Cordova 3107, Vitacura, Casilla 19001, Santiago, Chile

32 European Space Agency, ESAC, Planck Science Office, Camino bajo del Castillo, s/n, Urbanización Villafranca del Castillo, Villanueva de la Cañada, Madrid, Spain

33 European Space Agency, ESTEC, Keplerlaan 1, 2201 AZ Noordwijk, The Netherlands

34 Haverford College Astronomy Department, 370 Lancaster Avenue, Haverford, Pennsylvania, USA

35 Helsinki Institute of Physics, Gustaf Hällströmin katu 2, University of Helsinki, Helsinki, Finland

36 INAF - Osservatorio Astrofisico di Catania, via S. Sofia 78, Catania, Italy

37 INAF - Osservatorio Astronomico di Padova, vicolo dell'Osservatorio 5, Padova, Italy

38 INAF - Osservatorio Astronomico di Roma, via di Frascati 33, Monte Porzio Catone, Italy

39 INAF - Osservatorio Astronomico di Trieste, via G.B. Tiepolo 11 , Trieste, Italy

40 INAF/IASF Bologna, via Gobetti 101, Bologna, Italy

41 INAF/IASF Milano, via E. Bassini 15, Milano, Italy

42 INRIA, Laboratoire de Recherche en Informatique, Université Paris-Sud 11, Bâtiment 490, 91405 Orsay Cedex, France

43 Imperial College London, Astrophysics group, Blackett Laboratory, Prince Consort Road, London, SW7 2AZ, UK

44 Infrared Processing and Analysis Center, California Institute of Technology, Pasadena, CA 91125, USA

45 Institut Néel, CNRS, Université Joseph Fourier Grenoble I, 25 rue des Martyrs, Grenoble, France

46 Institut d'Astrophysique Spatiale, CNRS (UMR8617) Université Paris-Sud 11, Bâtiment 121, Orsay, France

47 Institut d'Astrophysique de Paris, CNRS UMR7095, Université Pierre \& Marie Curie, 98 bis boulevard Arago, Paris, France

48 Institute of Astronomy and Astrophysics, Academia Sinica, Taipei, Taiwan

49 Institute of Astronomy, University of Cambridge, Madingley Road, Cambridge CB3 0HA, UK

50 Institute of Theoretical Astrophysics, University of Oslo, Blindern, Oslo, Norway

51 Instituto de Astrofísica de Canarias, C/Vía Láctea s/n, La Laguna, Tenerife, Spain

52 Instituto de Física de Cantabria (CSIC-Universidad de Cantabria), Avda. de los Castros s/n, Santander, Spain

53 Istituto di Fisica del Plasma, CNR-ENEA-EURATOM Association, via R. Cozzi 53, Milano, Italy

54 Jet Propulsion Laboratory, California Institute of Technology, 4800 Oak Grove Drive, Pasadena, California, USA

55 Jodrell Bank Centre for Astrophysics, Alan Turing Building, School of Physics and Astronomy, The University of Manchester, Oxford Road, Manchester, M13 9PL, UK

56 Kavli Institute for Cosmology Cambridge, Madingley Road, Cambridge, CB3 0HA, UK

57 LERMA, CNRS, Observatoire de Paris, 61 Avenue de l'Observatoire, Paris, France

58 Laboratoire AIM, IRFU/Service d'Astrophysique - CEA/DSM CNRS - Université Paris Diderot, Bât. 709, CEA-Saclay, 91191 Gif-sur-Yvette Cedex, France 
59 Laboratoire Traitement et Communication de l'Information, CNRS (UMR 5141) and Télécom ParisTech, 46 rue Barrault 75634 Paris Cedex 13, France

${ }^{60}$ Laboratoire de Physique Subatomique et de Cosmologie, CNRS/IN2P3, Université Joseph Fourier Grenoble I, Institut National Polytechnique de Grenoble, 53 rue des Martyrs, 38026 Grenoble Cedex, France

${ }^{61}$ Laboratoire de l'Accélérateur Linéaire, Université Paris-Sud 11, CNRS/IN2P3, Orsay, France

62 Lawrence Berkeley National Laboratory, Berkeley, California, USA

${ }^{63}$ Max-Planck-Institut für Astrophysik, Karl-Schwarzschild-Str. 1, 85741 Garching, Germany

64 Max-Planck-Institut für Radioastronomie, Auf dem Hügel 69, 53121 Bonn, Germany

65 MilliLab, VTT Technical Research Centre of Finland, Tietotie 3, Espoo, Finland

${ }^{66}$ National University of Ireland, Department of Experimental Physics, Maynooth, Co. Kildare, Ireland

67 Niels Bohr Institute, Blegdamsvej 17, Copenhagen, Denmark

68 Observational Cosmology, Mail Stop 367-17, California Institute of Technology, Pasadena, CA, 91125, USA

69 Optical Science Laboratory, University College London, Gower Street, London, UK
70 SISSA, Astrophysics Sector, via Bonomea 265, 34136, Trieste, Italy

71 SUPA, Institute for Astronomy, University of Edinburgh, Royal Observatory, Blackford Hill, Edinburgh EH9 3HJ, UK

72 School of Physics and Astronomy, Cardiff University, Queens Buildings, The Parade, Cardiff, CF24 3AA, UK

73 Space Sciences Laboratory, University of California, Berkeley, California, USA

74 Spitzer Science Center, 1200 E. California Blvd., Pasadena, California, USA

75 Stanford University, Dept of Physics, Varian Physics Bldg, 382 via Pueblo Mall, Stanford, California, USA

76 Universidad de Chile, Casilla 36-D, Santiago, Chile

77 Université de Toulouse, UPS-OMP, IRAP, 31028 Toulouse Cedex 4, France

78 Universities Space Research Association, Stratospheric Observatory for Infrared Astronomy, MS 211-3, Moffett Field, CA 94035, USA

79 University of Granada, Departamento de Física Teórica y del Cosmos, Facultad de Ciencias, Granada, Spain

80 University of Miami, Knight Physics Building, 1320 Campo Sano Dr., Coral Gables, Florida, USA

81 Warsaw University Observatory, Aleje Ujazdowskie 4, 00-478 Warszawa, Poland 